\title{
Surgery
}

\section{Quantitative fluorescence angiography vs. hyperspectral imaging to assess bowel ischemia: a comparative study in enhanced reality \\ --Manuscript Draft--}

\begin{tabular}{|c|c|}
\hline Manuscript Number: & 20191302R1 \\
\hline Article Type: & Original Communication \\
\hline Section/Category: & Other \\
\hline Keywords: & $\begin{array}{l}\text { Fluorescence Imaging; quantitative fluorescence angiography; hyperspectral imaging; } \\
\text { imaging spectrometer; bowel perfusion; enhanced reality; local capillary lactate }\end{array}$ \\
\hline Corresponding Author: & $\begin{array}{l}\text { Manuel Barberio, MD } \\
\text { FRANCE }\end{array}$ \\
\hline First Author: & Manuel Barberio, MD \\
\hline \multirow[t]{11}{*}{ Order of Authors: } & Manuel Barberio, MD \\
\hline & Eric Felli \\
\hline & Emilie Seyller, MD \\
\hline & Fabio Longo, MD \\
\hline & Manish Chand, MD, PhD \\
\hline & Ines Gockel, MD, MBA \\
\hline & Bernard Geny, MD, PhD \\
\hline & Lee Swanström, MD, PhD \\
\hline & Jacques Marescaux, MD, FACS \\
\hline & Vincent Agnus, PhD \\
\hline & Michele Diana, MD, PhD \\
\hline Manuscript Region of Origin: & FRANCE \\
\hline Abstract: & $\begin{array}{l}\text { Background } \\
\text { FLuorescence-based Enhanced Reality (FLER) is a software providing quantitative } \\
\text { fluorescence angiography (FA), by computing the fluorescence intensity time-to-peak } \\
\text { (TTP), after intravenous injection of indocyanine green (ICG). } \\
\text { HSI is a contrast-free optical imaging modality, which measures tissue oxygenation } \\
\text { (StO } 2 \text { ). } \\
\text { Methods } \\
\text { In } 8 \text { pigs, an ischemic bowel segment was created by dividing the arcade branches } \\
\text { and imaged using HSI and FLER. StO } 2 \text { values were acquired through a } \\
\text { hyperspectral imaging (HSI) system. Subsequently, FA was performed using a near- } \\
\text { infrared laparoscopic camera, after intravenous injection of } 0.2 \mathrm{mg} / \mathrm{kg} \text { of ICG. The TTP } \\
\text { fluorescence signal was analyzed through a proprietary software to realize a perfusion } \\
\text { map. This was overlaid onto real-time images to obtain FLER. Simultaneously, nine } \\
\text { adjacent regions of interest (ROIs) were selected and superimposed onto the real-time } \\
\text { video obtaining HYperspectral-based Enhanced Reality (HYPER). FLER and HYPER } \\
\text { were superimposed allowing a comparison of both imaging modalities. Local capillary } \\
\text { lactates (LCL) were sampled at the ROIs. Two LCL prediction models were } \\
\text { extrapolated based on both imaging. } \\
\text { Results } \\
\text { For all ROIs mean LCL measured } 4.67 \pm 4.34 \text { mmol/L, mean StO } 245.92 \pm 18.59 \% \text {, } \\
\text { and mean TTP } 10.33 \pm 9.36 \text { sec. Pearson's test between FLER-TTP and HSI-StO2 at } \\
\text { the corresponding ROls gave an R=-0.66 ( } p<0.0001) \text {. The HSI lactate prediction model } \\
\text { proved significantly more accurate than the FLER-based one ( } p<0.0001) \text {. }\end{array}$ \\
\hline
\end{tabular}




\section{Conclusion}

Bowel perfusion was clearly quantified using $\mathrm{HSI}$ and FA. HSI yielded more accurate results than FA. HYPER might become a useful intraoperative tool to quantify bowel ischemia in a contrast-free fashion. 
Quantitative fluorescence angiography vs. hyperspectral imaging to assess bowel ischemia: a comparative study in enhanced reality

Manuel Barberio ${ }^{1,3,4}$, MD; Eric Felli ${ }^{1}$; Emilie Seyller ${ }^{1}$, MD; Fabio Longo ${ }^{1}$, MD; Manish Chand $^{2}$, MD, PhD; Ines Gockel ${ }^{3}$, MD, MBA; Bernard Geny ${ }^{4}, \mathrm{MD}, \mathrm{PhD}$; Lee Swanström ${ }^{1}, \mathrm{MD}$, PhD; Jacques Marescaux ${ }^{1,5}$, MD, FACS, (Hon) FRCS, (Hon) FJSES, (Hon) FASA; Vincent Agnus $^{1}, \mathrm{PhD}$; Michele Diana ${ }^{1,4}, \mathrm{MD}, \mathrm{PhD}$

1) IHU-Strasbourg Institute of Image-Guided Surgery, Strasbourg, France

2) Division of Surgery \& Interventional Science, University College London, London, UK

3) Department of Visceral, Transplant, Thoracic and Vascular Surgery, University Hospital of Leipzig, Leipzig, Germany

4) EA 3072, Fédération de Médecine Translationnelle de Strasbourg, Medical University of Strasbourg, Strasbourg, France

5) Research Institute against Digestive Cancer (IRCAD), Strasbourg, France

\section{Corresponding author:}

Manuel Barberio, MD

IHU-Strasbourg, Institute of Hybrid Image-Guided Surgery, Strasbourg, France

1, place de l'Hôpital, 67091 Strasbourg, France

Email : manuel.barberio@ihu-strasbourg.eu

Phone: +33768281308

A partial overview of this work was presented at the 27th European Association for Endoscopic Surgery (EAES) congress in Seville, Spain, June 12-15, 2019. 


\section{Abstract}

\section{Background}

FLuorescence-based Enhanced Reality (FLER) is a software providing quantitative fluorescence angiography (FA), by computing the fluorescence intensity time-to-peak (TTP), after intravenous injection of indocyanine green (ICG).

HSI is a contrast-free optical imaging modality, which measures tissue oxygenation $\left(\mathrm{StO}_{2}\right)$.

\section{Methods}

In 8 pigs, an ischemic bowel segment was created by dividing the arcade branches and imaged using HSI and FLER. $\mathrm{StO}_{2}$ values were acquired through a hyperspectral imaging (HSI) system. Subsequently, FA was performed using a near-infrared laparoscopic camera, after intravenous injection of $0.2 \mathrm{mg} / \mathrm{kg}$ of ICG. The TTP fluorescence signal was analyzed through a proprietary software to realize a perfusion map. This was overlaid onto real-time images to obtain FLER. Simultaneously, nine adjacent regions of interest (ROIs) were selected and superimposed onto the real-time video obtaining HYperspectral-based Enhanced Reality (HYPER). FLER and HYPER were superimposed allowing a comparison of both imaging modalities. Local capillary lactates (LCL) were sampled at the ROIs. Two LCL prediction models were extrapolated based on both imaging.

\section{Results}

For all ROIs mean LCL measured 4.67 $\pm 4.34 \mathrm{mmol} / \mathrm{L}$, mean $\mathrm{StO}_{2} 45.92 \pm 18.59 \%$, and mean TTP $10.33 \pm 9.36 \mathrm{sec}$. Pearson's test between FLER-TTP and HSI-StO2 at the corresponding ROIs gave an $\mathrm{R}=-0.66(\mathrm{p}<0.0001)$. The HSI lactate prediction model proved significantly more accurate than the FLER-based one $(\mathrm{p}<0.0001)$.

\section{Conclusion}

Bowel perfusion was clearly quantified using HSI and FA. HSI yielded more accurate results than FA. HYPER might become a useful intraoperative tool to quantify bowel ischemia in a contrast-free fashion.

Keywords: fluorescence imaging, quantitative fluorescence angiography, hyperspectral imaging, imaging spectrometer, bowel perfusion, enhanced reality, local capillary lactate. 


\section{Background}

The incidence of gastrointestinal anastomotic leakage (AL) remains high, ranging from 20 to $35 \%^{1}$ after esophageal procedures, and from 4 to $19 \%^{2}$ after colorectal procedures. Additionally, AL is associated with high morbidity and mortality rates ${ }^{3}$.

Inadequate perfusion to the bowel ends being anastomosed remains the most important determinant of anastomotic breakdown ${ }^{3}$. The clinical intraoperative evaluation of gastrointestinal perfusion is unreliable irrespective of the surgeon's experience ${ }^{4}$.

Fluorescence angiography (FA) is a real-time optical imaging technique, which allows to estimate bowel perfusion through the enhanced visualization of an intravenously injected fluorescent dye, mostly indocyanine green (ICG) using a Near-Infrared (NIR) camera system. Several clinical trials have pointed out that FA holds some potential in preventing $\mathrm{AL}^{5-9}$. However, a standardized protocol for FA and a quantitative metric of the fluorescent signal are currently missing ${ }^{10}$.

Fluorescence-based Enhanced Reality (FLER) is a software solution, which computes the timeto-peak (TTP) of the fluorescent signal pixel-by-pixel during FA. The accuracy of the quantification software has been validated in the large animal model using robust biological perfusion markers, including local capillary lactates (LCL), mitochondrial respiration and metabolomics $^{11-14}$.

Hyperspectral imaging (HSI) is another optical imaging technique, which combines a spectroscope and a photo camera, allowing for a contrast-free, real-time, qualitative and quantitative tissue analysis based on tissue oxygen saturation $\left(\mathrm{StO}_{2}\right)^{15,16}$. The downside of HSI, at the present stage of development, is in the lack of a video rate and the absence of a minimally invasive device. Through a customization of the HSI system and a proprietary software, static HSI perfusion images can be superimposed onto a real-time video, in order to generate an HSIpowered enhanced reality (HYPER) environment, allowing for a precise surgical navigation. 
The accuracy of HYPER in quantifying from early (5 minutes) to late (360 minutes) bowel ischemia using reliable cell suffering surrogates, such as LCL and reactive oxygen species (ROS) production, has been previously demonstrated ${ }^{17}$. The aim of the present study was to compare the accuracy of FLER and HYPER in a porcine, non-survival, open surgery model of bowel ischemia. 


\section{Methods}

Animal characteristics

Eight adult male pigs (Large White, mean weight: $36.6 \pm 6.2 \mathrm{~kg}$ ) were included in the present study, which is part of the ELIOS project (Endoscopic Luminescent Imaging for Oncology Surgery), approved by the local Ethical Committee on Animal Experimentation (ICOMETH No. 38.2016.01.085), and by the French Ministry of Superior Education and Research (MESR) (APAFIS\#8721-2017013010316298-v2). Only male sex was chosen in order to have a homogeneous subjects' sample. All animals were managed according to French laws for animal use and care, and according to the directives of the European Community Council (2010/63/EU) and ARRIVE guidelines ${ }^{18}$.

The animals were fasted for 24 hours with free access to water before surgery. Premedication was administered 10 minutes before surgery, using an intramuscular injection of ketamine $(20 \mathrm{mg} / \mathrm{kg})$ and azaperone $(2 \mathrm{mg} / \mathrm{kg})$ (Stresnil, Janssen-Cilag, Belgium). Intravenous propofol $(3 \mathrm{mg} / \mathrm{kg})$ combined with rocuronium $(0.8 \mathrm{mg} / \mathrm{kg})$ was used for induction. Anesthesia was maintained with $2 \%$ isoflurane. At the end of the procedures, pigs were sacrificed with an intravenous injection of Pentobarbital Sodium $(40 \mathrm{mg} / \mathrm{kg}$ ) (Exagon®, AXIENCE, France), under 5\% isoflurane anesthesia.

\section{Hyperspectral-based enhanced reality}

A CMOS hyperspectral push-broom imaging system (TIVITA®, Diaspective Vision GmbH, Germany) was used; the camera lens was placed at a distance of $35 \mathrm{~cm}$ from the surgical scene. The HSI camera is able to quantify $\mathrm{StO}_{2}$ by generating pseudo-color images as an instantaneous output (acquisition time: roughly $6 \mathrm{sec}$ ), by means of the integrated software. As previously described $^{17}$, the hardware was customized, by adding an aligned video-camera and an infrared 
distance sensor. A Python ${ }^{\mathrm{TM}}$-based proprietary software allowed to superimpose the $\mathrm{StO}_{2}$ quantification images onto the real-time video of the surgical field, and HYPER was obtained.

\section{Fluorescence-based Enhanced Reality}

After an intravenous injection of $0.2 \mathrm{mg} / \mathrm{kg}$ Indocyanine Green (ICG) (Infracyanine ${ }^{\circledR}$, Serb, Paris, France), the fluorescent signal was captured with a 30-degree near-infrared laparoscope (D-Light P, KARL STORZ Endoscope, Tuttlingen, Germany), placed at the shortest distance required to capture the entire surgical area of interest. The ER-PERFUSION software (IRCAD, France) computes perfusion as the TTP of the fluorescent signal evolution pixel-by-pixel.

TTP is converted into a quantitative perfusion cartography, generated by analyzing the velocity of the fluorescence signal until it reaches its maximal intensity peak. The perfusion cartography is a color-coded image, which can be used as a last image hold function and overlaid onto the real-time laparoscopic video. Fluorescence intensity varies largely depending on the distance between the NIR camera and the target. Since we used the relative fluorescence signal variation over time, our measure was not influenced by distance. TTP is obtained as a difference $\mathrm{T}_{75^{-}} \mathrm{T}_{25}$, where $T_{25}$ and $T_{75}$ represent timepoints corresponding to the first and last quartile of fluorescent intensity over time respectively (Figure 1). The difference between $\mathrm{T}_{100}$ (maximum intensity of fluorescent signal) and $\mathrm{T}_{0}$ (minimum intensity of fluorescent signal), has been previously used to compute $\mathrm{TTP}^{19}$. However, it is often difficult to precisely determine the timepoints of the minimum (given the frequent presence of signal noise) and maximum (given the presence of a long signal plateau phase) fluorescence intensity. As a result, $\mathrm{T}_{25}$ and $\mathrm{T}_{75}$ were chosen, since they are more robust timepoints.

An ICG reference card (Green Balance ${ }^{\mathrm{TM}}$, Diagnostic Green GmbH, Aschheim-Dornach, Germany) was placed in the surgical field during FLER acquisitions in order to test the fluorescence signal before ICG injection. 
The software generated a quantitative perfusion map, which was superimposed onto the laparoscopic real-time video to obtain FLER, as previously described ${ }^{11-14}$ (Figure 2).

\section{Capillary lactate analysis}

Using the level of local capillary lactates (LCL) as primary outcome, a sample size calculation was performed based on previous works from our group, using a similar experimental model. Using a superiority design, 50 simultaneously analyzed spots with both techniques were required to have a $90 \%$ chance of detecting, as significant at the $5 \%$ level, a difference in the primary outcome measure. Since an ischemic bowel loop was created in each animal and each loop contained 9 ROIs, 8 animals (72 ROIs) in total were included.

LCL served as "ground-truth" metric to compare FLER and HYPER performances. A stripbased, portable lactate analyzer (EDGE®, ApexBio, Taipei, Taiwan; measuring range: 1.1$22.2 \mathrm{mmol} / \mathrm{L}$ ) was used to assess lactate levels on blood punctured from the serosal capillary vessels in correspondence with each identified ROI. The order of sampling was randomized. The robustness of LCL to quantify the perfusion of the gastrointestinal tract has been largely demonstrated in the past ${ }^{11-14,17,20,21}$.

Systemic lactatemia was measured on blood samples obtained by puncturing capillary vessels on the pigs' snout and LCL were normalized to the systemic values.

\section{Surgical set-up and experimental workflow}

A laparotomy was performed, and a $10 \mathrm{~cm}$ ischemic segment was created in the proximal jejunum, by dividing the arcade branches (Figure 3). After 30 minutes of ischemia, HYPER was first performed, displaying the $\mathrm{StO}_{2}$ pseudo-color images onto the real-time laparoscopic video shown directly on the OR monitor. Nine contiguous regions of interest (ROI), including perfused and ischemic areas, were randomly placed throughout the length of the bowel loop 
and displayed in augmented reality. Immediately afterwards, ICG was administered intravenously, and FLER was obtained and simultaneously superimposed onto HYPER (Figure 2). As next step, the sampling of LCL at the 9 ROIs displayed in augmented reality was performed in randomized order.

The HSI images are obtained in approximately 6 seconds for each acquisition and the $\mathrm{StO}_{2}$ cartography is provided instantaneously. The time required to position the ROIs and obtain HYPER is approximately 1 minute. The delay between ICG administration, in order to perform FA and superimposition of FLER onto HYPER is approximately of 2 minutes. Therefore, the two imaging techniques were performed with a time interval of 1 minute. The delay between HYPER completion and lactate sampling was of roughly 3 minutes.

In order to minimize any bias, possibly generated by motion or light artefacts, breathing was stopped, and external light interference was prevented during the time required to obtain both imaging acquisitions (6 seconds for HSI and 40 seconds for FA).

\section{Prediction of local capillary lactates from FLER TTP and HSI StO2}

The relationship between LCL and FLER TTP and $\mathrm{HSI} \mathrm{StO}_{2}$ respectively, followed an exponential trend, which was modeled by an exponential regression analysis. This allowed us to generate a prediction algorithm of the LCL values from the corresponding FLER TTP and $\mathrm{HSI}-\mathrm{StO}_{2}$. The following fitting functions were found:

1) based on the FLER TTP:

predicted $L C L=e^{0.01 * T T P-0.553}+$ SystemicLactates

2) based on $\mathrm{HSI}-\mathrm{StO}_{2}$ :

predictedLCL $=e^{-0.049 * \mathrm{StO}_{2}+2.22}+$ SystemicLactates 
In order to validate the prediction models a mean square minimization of the objective function $\exp (-a x+b)$ was performed on normalized lactates, using the Scipy, Python library ${ }^{22}$. The prediction functions were obtained from the whole datasets, since no significative difference in terms of error prediction could be observed when performing a leave-one-(pig) out crossvalidation.

The accuracy of both models, indicated as the absolute difference between the sampled LCL values and the predicted LCL values of both prediction models, was computed on the whole data by the corresponding exponential equation.

\section{Statistical analysis}

Data are shown as mean \pm SD unless indicated otherwise. Statistics were performed using the Python Scikit-learn library ${ }^{23}$. A Pearson's test was used to compare variables showing a linear relationship. Spearman correlation and exponential regression were used to investigate variables presenting a non-linear relationship. A Wilcoxon test was performed for paired comparison of the lactate prediction algorithms (based on HYPER and on FLER), since a nonGaussian data distribution was observed. A $p$ value $<0.05$ was considered statistically significant. 


\section{Results}

The enhanced reality allowed to precisely identify the ROIs on the bowel loops $(n=72)$ as demonstrated by both HSI-StO2 and FLER TTP, enabling accurate sampling of serosal capillary blood to measure LCL. Mean systemic lactate concentration was $2.63 \pm 2.85 \mathrm{mmol} / \mathrm{L}$. Considering all the ROIs, the mean LCL concentration was $4.67 \pm 4.34 \mathrm{mmol} / \mathrm{L}$, the mean normalized LCL value was $2.034 \pm 2.44 \mathrm{mmol} / \mathrm{L}$, the mean $\mathrm{StO}_{2}$ value was $45.92 \pm 18.59 \%$ and the mean TTP was $10.33 \pm 9.36$ sec.

\section{Correlation between FLER TTP and HSI-StO2}

The Pearson analysis between FLER TTP and HSI-StO2 in correspondence with the same ROIs gave a $\mathrm{R}=-0.66(\mathrm{p}<0.0001)($ Figure $4 \mathbf{a})$.

Correlation between LCL and TTP / LCL and StO2

The Spearman correlation between LCL values and FLER TTP gave a $R=0.40(p=0.001)$, and a $\mathrm{R}=-0.62(\mathrm{p}<0.0001)$ between LCL values and HSI-StO2. Both correlations produced 7 outliers (Figure 4 b-c), including only completely ischemic ROIs, with LCL > 6 mmol/l. These 7 outliers were excluded from the analysis of the LCL prediction models accuracy reported below.

\section{LCL prediction based on FLER TTP}

The mean error of the lactate prediction model was $0.95 \pm 0.74 \mathrm{mmol} / \mathrm{L}$. The median error was $0.68 \mathrm{mmol} / \mathrm{L} .95 \%$ of the errors occur for lactate values $<2.33 \mathrm{mmol} / \mathrm{L}$.

\section{LCL prediction based on HSI-StO2}

The lactate prediction model showed a mean error of $0.65 \pm 0.59 \mathrm{mmol} / \mathrm{L}$, with a median error of $0.43 \mathrm{mmol} / \mathrm{L} .95 \%$ of the errors occur for lactate values $<2 \mathrm{mmol} / \mathrm{L}$.

\section{Comparison between FLER-based and HSI-based lactate prediction models}


The Wilcoxon test showed a significant difference between the two prediction models ( $\mathrm{p}<0.0001$ ), with HSI yielding a better lactate prediction performance (Figure 5). 


\section{Discussion}

The results of the present study show that both FLER and HYPER give virtually real-time information, and the metrics used by both quantitative methods, namely TTP and $\mathrm{StO}_{2}$, are significantly correlated. Additionally, both parameters have a significant exponential relationship with the capillary lactates. A fitting function between LCL and both the FLER TTP and $\mathrm{HSI}-\mathrm{StO}_{2}$ was found and provided two LCL prediction models. It was observed that both prediction models were less accurate for LCL values $>6 \mathrm{mmol} / \mathrm{L}$. As a result, a threshold below this value was set, and led to excluding a total of 7 ROIs which were definitely outliers (Figure $4 \mathbf{b - c})$. This correction lead to a substantially improved precision of the prediction algorithms. This finding is in line with the results of our prior study, in which HYPER provided a significantly better accuracy of the LCL prediction for $\mathrm{StO}_{2}$ values $>30 \%{ }^{17}$. In the present study, all outlying ROIs (LCL $>6 \mathrm{mmol} / \mathrm{L}$ ) showed that $\mathrm{StO}_{2}$ was $<30 \%$. Likewise, it is clinically irrelevant to precisely predict the LCL in ROIs which are frankly ischemic based on a quantitative optical imaging analysis and which are often identifiable as non-perfused to the naked eye. Both prediction models showed high accuracy in discriminating marginally perfused areas, which are generally difficult to locate under white light. The precise identification of those areas is important not only to assess the perfusion of gastrointestinal anastomoses, but also to evaluate the bowel viability after mesenteric ischemia. As outlined by Kougias et al., second-look laparotomies with additional bowel resections are required in $57 \%$ of cases presenting with mesenteric ischemia ${ }^{24}$. Therefore, non-invasive optical imaging tools, like FLER or HYPER, could improve the intraoperative decision-making process. However, the threshold of those imaging modalities representing the "point of no return" of bowel viability has yet to be identified and is currently under investigation.

The prediction model based on the FLER TTP was significantly less accurate when compared to the $\mathrm{HSI} \mathrm{StO}_{2}$ based model. A possible explanation could be the short ischemia period (30 
min) chosen in the present set-up. During this time, macroscopic signs of tissue suffering are

rarely found. However, there are intracellular changes which provoke a reduced mitochondrial activity. As a result, reduced $\mathrm{O}_{2}$ and increased LCL production already occur ${ }^{14}$. The HSIdetected $\mathrm{StO}_{2}$ seems to better mirror those intracellular metabolic changes as compared to FLER, which finally measures a macroscopic phenomenon such as the serosal surface blood flow.

FA is a well-established intraoperative method to assess gastrointestinal perfusion and is relatively easy to integrate in the surgical workflow ${ }^{9}$. However, there is still no consensus on the impact of FA on reducing the incidence of $\mathrm{AL}$ and the results of randomized clinical trials are awaited ${ }^{25}$. Two factors affect the performances of FA and should be controlled. The first one is the progressive diffusion of the fluorophore through the serosal capillary network into the margins of the ischemic zones over time, leading to an overestimation of the vascularized area. The second one is the quadratic inverse relationship between the target-endoscope distance and fluorescence intensity ${ }^{11,14}$. When distance is not controlled and/or fluorescence intensity is not normalized with a calibration system, the presence of a fluorescent signal in the tissue is not representative of the true perfusion. To overcome such interpretation biases, a software-based quantitative FA was introduced, based on the analysis of fluorescent signal dynamics $^{11,14,19}$. Wada et al. successfully created an algorithm of quantitative fluorescence analysis during clinical colorectal cases ${ }^{19}$. The authors used a similar algorithm of fluorescence dynamics evaluation ${ }^{11}$. However, unlike FLER, the enhanced information (virtual perfusion cartography) was not superimposed onto real-time images. FLER is also currently being evaluated in a clinical trial ${ }^{26,}{ }^{27}$ (https://clinicaltrials.gov/ct2/show/NCT02626091) with promising early results in terms of correlation with surrogate markers of perfusion and prediction of anastomotic complications. 
HSI has been used for decades in various industrial sectors or for remote sensing ${ }^{28}$. Recently,

HSI was successfully used in medical and surgical applications, including tumor identification $^{28}$, intraoperative parathyroid recognition ${ }^{29}$, and real-time quantitative perfusion assessment during gastrointestinal surgical procedures $\mathrm{s}^{30-32}$.

Compared to fluorescence imaging, HSI provides a larger amount of information and is able to quantify several tissue components, including water, oxygen, and hemoglobin content. Additionally, a remarkable advantage when compared to FA is that HSI does not require the use of any contrast agents. In other words, HSI is an optical, contrast-free, and non-destructive in vivo "biopsy" of the surgical field, allowing one to virtually discriminate anatomical structures and characterize tissue physiology. However, the large HSI-generated datasets require complex data processing algorithms in order to extrapolate useful discriminative features from the spectral curves. The TIVITA® HSI system has a built-in software, which generates pseudo-color images, quantifying $\mathrm{StO}_{2}{ }^{29-31}$. We used a proprietary software solution for the superimposing of the $\mathrm{HSI} \mathrm{StO}_{2}$ images onto real-time video images in order to create an enhanced reality surgical scene (HYPER) and precisely identify the small ROIs on the bowel surface.

To the best of our knowledge, the present study is the first comparison between HSI and quantitative FA to assess bowel perfusion. The strong point of our study lies in its robust design and methodology. Limitations lie in the fact that the results are obtained in controlled experimental conditions of early ischemia (30 minutes) and the clinical application of HYPER has not been demonstrated yet. Furthermore, although the acquisitions of the two imaging systems occurred virtually simultaneously (1-minute delay), the study draws on comparison between a minimally invasive NIR camera and an open-surgery HSI camera. In this setting, HYPER displayed a significantly better overall accuracy. While this difference was statistically significant, it remains unknown if it would prove to be clinically significant. 
However, at the moment, HSI systems are limited by the lack of an adequately fast video rate and by the lack of a minimally invasive surgery system. Some authors have proposed endoscopic HSI systems $s^{33,34}$. However, the spatial resolution and data processing speed are still limited to prevent daily surgical practice implementation.

At the moment, HYPER represents a good tool in order to perform a non-invasive, contrastfree, real-time and accurate, intraoperative perfusion quantification.

As next step, a trial to assess the accuracy of HYPER intraoperatively is currently being designed.

\section{Conclusions}

In the acute experimental setting, quantitative evaluation of perfusion using hyperspectral imaging was more accurate when compared to quantitative fluorescence-based evaluation. The next step is clinical translation and the evaluation of the impact of those emerging technologies on the incidence of anastomotic leakage.

\section{Acknowledgments}

Authors are grateful to Guy Temporal, Christopher Burel, and Camille Goustiaux, professionals in medical English proofreading, for their valuable help in revising the manuscript.

Disclosures: Jacques Marescaux is the President of both IRCAD and IHU Strasbourg Institutes, which are partly funded by KARL STORZ, Siemens, and Medtronic. Lee Swanström is the Scientific Director of the IHU and Michele Diana is the recipient of the ELIOS grant. Manish Chand has received fees from Stryker Endoscopy for his work as a consultant for fluorescent 
angiography, outside of this work. The remaining authors have no conflicts of interest or

\section{Funding source:}

This work was funded by the ARC Foundation through the ELIOS (Endoscopic Luminescent Imaging for precision Oncologic Surgery) grant.

\section{References:}

1. Campbell C, Reames MK, Robinson M, Symanowski J, Salo JC. Conduit Vascular Evaluation is Associated with Reduction in Anastomotic Leak After Esophagectomy. J Gastrointest Surg. 2015;19:806-12.

2. Karliczek A, Benaron DA, Baas PC, Zeebregts CJ, Wiggers T, van Dam GM. Intraoperative assessment of microperfusion with visible light spectroscopy for prediction of anastomotic leakage in colorectal anastomoses. Colorectal Dis.12:1018-25.

3. Urbanavicius L, Pattyn P, de Putte DV, Venskutonis D. How to assess intestinal viability during surgery: A review of techniques. World J Gastrointest Surg. 2011;3:59-69.

4. Karliczek A, Harlaar NJ, Zeebregts CJ, Wiggers T, Baas PC, van Dam GM. Surgeons lack predictive accuracy for anastomotic leakage in gastrointestinal surgery. Int J Colorectal Dis. 2009;24:569-76.

5. Kudszus S, Roesel C, Schachtrupp A, Hoer JJ. Intraoperative laser fluorescence angiography in colorectal surgery: a noninvasive analysis to reduce the rate of anastomotic leakage. Langenbecks Arch Surg. 2010;395:1025-30.

6. Jafari MD, Wexner SD, Martz JE, McLemore EC, Margolin DA, Sherwinter DA, et al. Perfusion assessment in laparoscopic left-sided/anterior resection (PILLAR II): a multiinstitutional study. J Am Coll Surg. 2015;220:82-92 e1.

7. Ris F, Liot E, Buchs NC, Kraus R, Ismael G, Belfontali V, et al. Multicentre phase II trial of near-infrared imaging in elective colorectal surgery. Br J Surg. 2018;105:1359-67.

8. De Nardi P, Elmore U, Maggi G, Maggiore R, Boni L, Cassinotti E, et al. Intraoperative angiography with indocyanine green to assess anastomosis perfusion in patients undergoing laparoscopic colorectal resection: results of a multicenter randomized controlled trial. Surg Endosc. 2019.

9. Blanco-Colino R, Espin-Basany E. Intraoperative use of ICG fluorescence imaging to reduce the risk of anastomotic leakage in colorectal surgery: a systematic review and meta-analysis. Tech Coloproctol. 2018;22:15-23.

10. Vallance A, Wexner S, Berho M, Cahill R, Coleman M, Haboubi N, et al. A collaborative review of the current concepts and challenges of anastomotic leaks in colorectal surgery. Colorectal Dis. 2017;19:01-012. 
11. Diana M, Agnus V, Halvax P, Liu YY, Dallemagne B, Schlagowski Al, et al. Intraoperative fluorescence-based enhanced reality laparoscopic real-time imaging to assess bowel perfusion at the anastomotic site in an experimental model. Br J Surg. 2015;102:e16976.

12. Diana $M$, Dallemagne $B$, Chung $H$, Nagao $Y$, Halvax $P$, Agnus $V$, et al. Probe-based confocal laser endomicroscopy and fluorescence-based enhanced reality for real-time assessment of intestinal microcirculation in a porcine model of sigmoid ischemia. Surg Endosc. 2014;28:3224-33.

13. Diana M, Halvax P, Dallemagne B, Nagao Y, Diemunsch P, Charles AL, et al. Real-time navigation by fluorescence-based enhanced reality for precise estimation of future anastomotic site in digestive surgery. Surg Endosc. 2014;28:3108-18.

14. Diana M, Noll E, Diemunsch P, Dallemagne B, Benahmed MA, Agnus V, et al. Enhancedreality video fluorescence: a real-time assessment of intestinal viability. Ann Surg. 2014;259:700-7.

15. Grambow E, Dau M, Holmer A, Lipp V, Frerich B, Klar E, et al. Hyperspectral imaging for monitoring of perfusion failure upon microvascular anastomosis in the rat hind limb. Microvasc Res. 2018;116:64-70.

16. Holmer A, Marotz J, Wahl P, Dau M, Kammerer PW. Hyperspectral imaging in perfusion and wound diagnostics - methods and algorithms for the determination of tissue parameters. Biomed Tech (Berl). 2018;63:547-56.

17. Barberio M, Longo F, Fiorillo C, Seeliger B, Mascagni P, Agnus V, et al. HYPerspectral Enhanced Reality (HYPER): a physiology-based surgical guidance tool. Surg Endosc. 2019.

18. Kilkenny C, Browne W, Cuthill IC, Emerson M, Altman DG, Group NCRRGW. Animal research: reporting in vivo experiments: the ARRIVE guidelines. J Gene Med. 2010;12:561-3.

19. Wada T, Kawada K, Takahashi R, Yoshitomi M, Hida K, Hasegawa S, et al. ICG fluorescence imaging for quantitative evaluation of colonic perfusion in laparoscopic colorectal surgery. Surg Endosc. 2017;31:4184-93.

20. Diana M, Noll E, Charles AL, Diemunsch P, Geny B, Liu YY, et al. Precision real-time evaluation of bowel perfusion: accuracy of confocal endomicroscopy assessment of stoma in a controlled hemorrhagic shock model. Surg Endosc. 2017;31:680-91.

21. Diana M, Noll E, Diemunsch P, Moussallieh FM, Namer IJ, Charles AL, et al. MetabolismGuided Bowel Resection: Potential Role and Accuracy of Instant Capillary Lactates to Identify the Optimal Resection Site. Surg Innov. 2015;22:453-61.

22. Virtanen P, Gommers R, Oliphant TE, Haberland M, Reddy T, Cournapeau D, et al. SciPy 1.0--Fundamental Algorithms for Scientific Computing in Python. arXiv preprint arXiv:190710121. 2019.

23. Pedregosa F, Varoquaux G, Gramfort A, Michel V, Thirion B, Grisel O, et al. Scikit-learn: Machine learning in Python. Journal of machine learning research. 2011;12:2825-30.

24. Kougias P, Lau D, El Sayed HF, Zhou W, Huynh TT, Lin PH. Determinants of mortality and treatment outcome following surgical interventions for acute mesenteric ischemia. Journal of vascular surgery. 2007;46:467-74.

25. Armstrong G, Croft J, Corrigan N, Brown JM, Goh V, Quirke P, et al. IntAct: intraoperative fluorescence angiography to prevent anastomotic leak in rectal cancer surgery: a randomized controlled trial. Colorectal Dis. 2018;20:0226-034. 
26. Seeliger B, Barberio M, D'Urso A, Agnus V, Longo F, Mascagni $P$, et al. Fluorescence in rectal cancer surgery. Annals of Laparoscopic and Endoscopic Surgery. 2018;3:47

27. Mascagni P, Longo F, Barberio M, Seeliger B, Agnus V, Saccomandi $P$, et al. New intraoperative imaging technologies: Innovating the surgeon's eye toward surgical precision. J Surg Oncol. 2018;118:265-82.

28. Lu G, Fei B. Medical hyperspectral imaging: a review. J Biomed Opt. 2014;19:10901.

29. Barberio M, Maktabi M, Gockel I, Rayes N, Jansen-Winkeln B, Köhler H, et al. Hyperspectral based discrimination of thyroid and parathyroid during surgery. Current Directions in Biomedical Engineering2018. p. 399.

30. Jansen-Winkeln B, Maktabi M, Takoh JP, Rabe SM, Barberio M, Kohler $H$, et al. [Hyperspectral imaging of gastrointestinal anastomoses]. Chirurg. 2018;89:717-25.

31. Kohler $H$, Jansen-Winkeln B, Maktabi M, Barberio M, Takoh J, Holfert $N$, et al. Evaluation of hyperspectral imaging (HSI) for the measurement of ischemic conditioning effects of the gastric conduit during esophagectomy. Surg Endosc. 2019.

32. Jansen-Winkeln B, Holfert N, Kohler H, Moulla $Y$, Takoh JP, Rabe SM, et al. Determination of the transection margin during colorectal resection with hyperspectral imaging (HSI). Int J Colorectal Dis. 2019;34:731-9.

33. Clancy NT, Arya S, Stoyanov D, Singh M, Hanna GB, Elson DS. Intraoperative measurement of bowel oxygen saturation using a multispectral imaging laparoscope. Biomed Opt Express. 2015;6:4179-90.

34. Zuzak KJ, Naik SC, Alexandrakis G, Hawkins D, Behbehani K, Livingston E. Intraoperative bile duct visualization using near-infrared hyperspectral video imaging. Am J Surg. 2008;195:491-7.

\section{Figures Legends}

Fig.1: TTP-representation. Graphical representation of the time-to-peak in a well perfused (blue) and in an ischemic area (green), in which very different maximum fluorescent intensity peaks (Fmax) are reached. TTP is the time required (in seconds) for the intensity curve to go from the first to the last quartile of fluorescent intensity over time. As represented, TTP is shorter for the perfused zone when compared to the ischemic one.

Fig.2: Enhanced reality modalities overlays. Ischemic small bowel model displayed under white light (A), with ICG reference card visible in the center of the bowel loop. FLER (B) with superimposing of the fluorescence-based quantitative perfusion cartography. HYPER (C) generated through the superimposing of the $\mathrm{StO}_{2}$ pseudo-color image captured with HSI. (D) shows the warping of FLER and HYPER simultaneously, over the real-time video captured 
with the NIR-laparoscopic camera during the white light mode. The selected ROIs are precisely identifiable in the surgical scene and LCL were sampled in correspondence with them.

Figure 3: Surgical set-up. The ischemic loop is placed on top of the calibration chessboard in order to facilitate the accurate superimposing of images obtained from cameras with different angles. The laparoscopic camera (arrow) and the HSI system (arrowhead) are placed in order to capture the surgical scene from different angles but with the same picture orientation.

Figure 4: $\mathrm{TTP}_{\mathrm{StO}}$ correlation and LCL regression analysis. (A) Visual representation of Pearson's correlation between HSI-StO2 and FLER TTP. The exponential regression correlation between lactates and both FLER TTP (B) and HSI-StO2 (C) is shown. Seven datasets of highly ischemic regions (shadowed points) were excluded from the regression analysis, since it was observed that, using both imaging modalities, the LCL prediction models are less accurate when $\mathrm{LCL}>6 \mathrm{mmol} / \mathrm{L}$, as highlighted in the graph.

Figure 5: Cumulative error of lactate prediction. Overall precision in predicting lactates is significantly higher when using the HSI-StO2 (black line) when compared to FLER TTP (red line). 


\section{Time-to-peak representation}

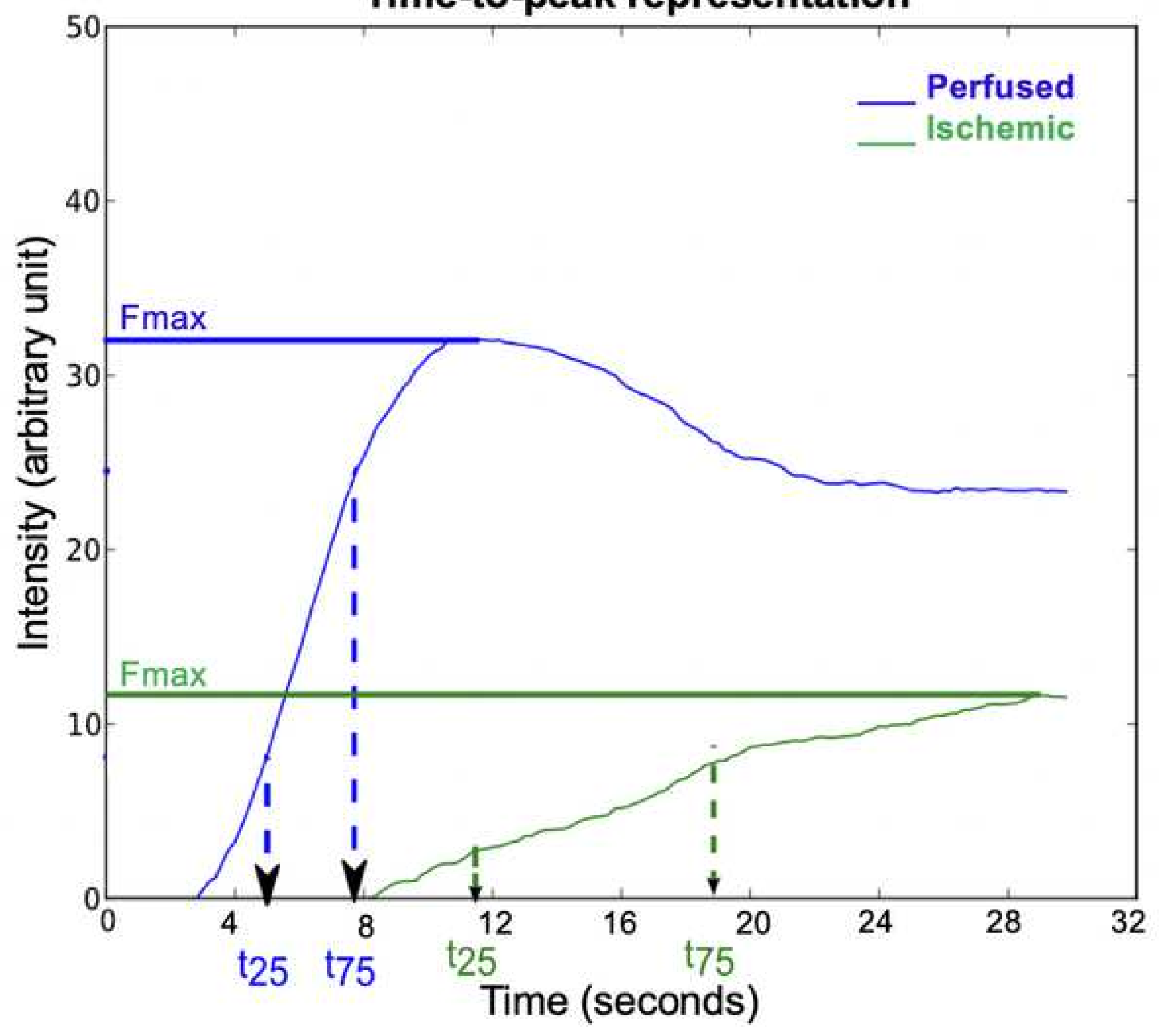




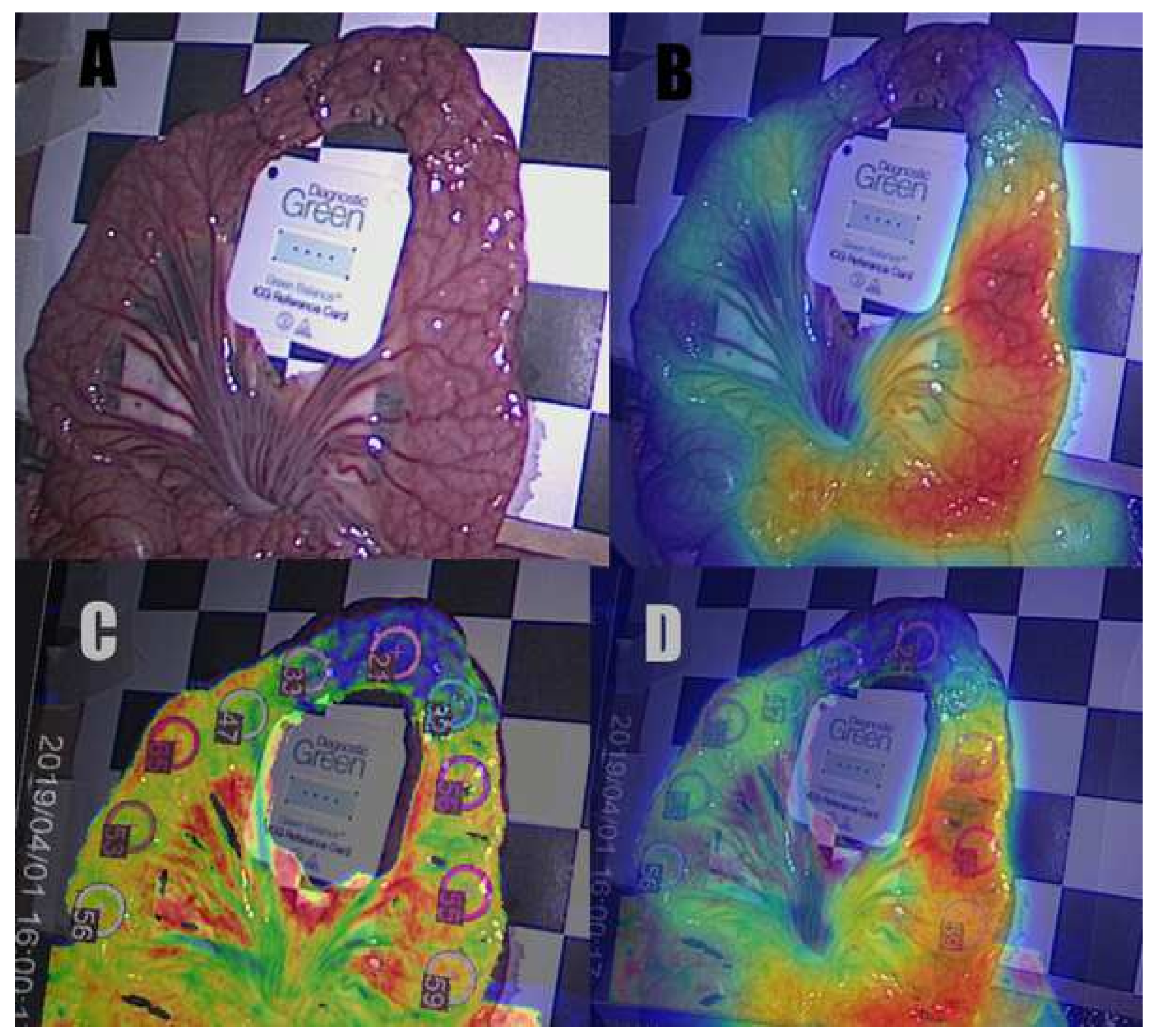




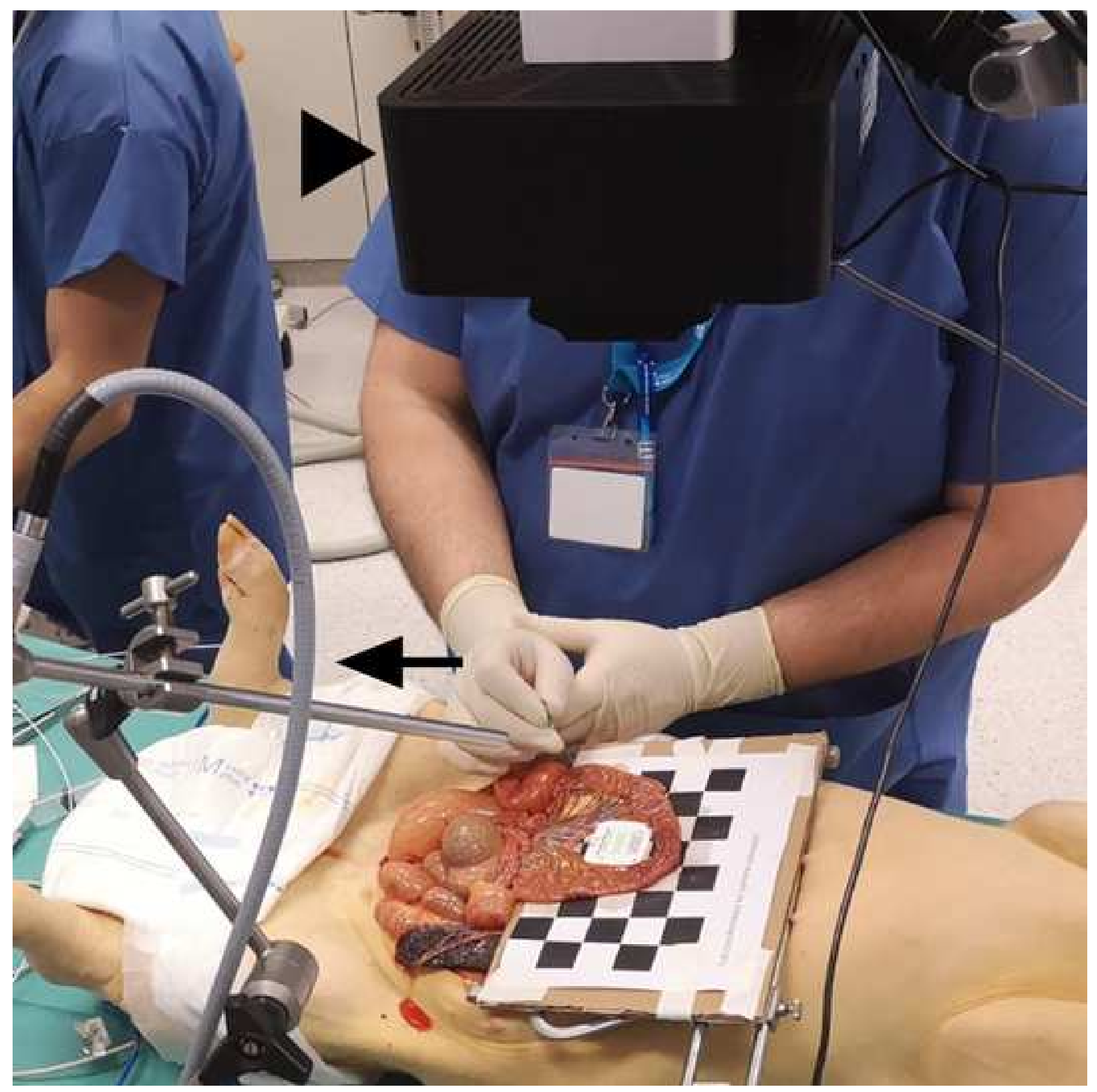



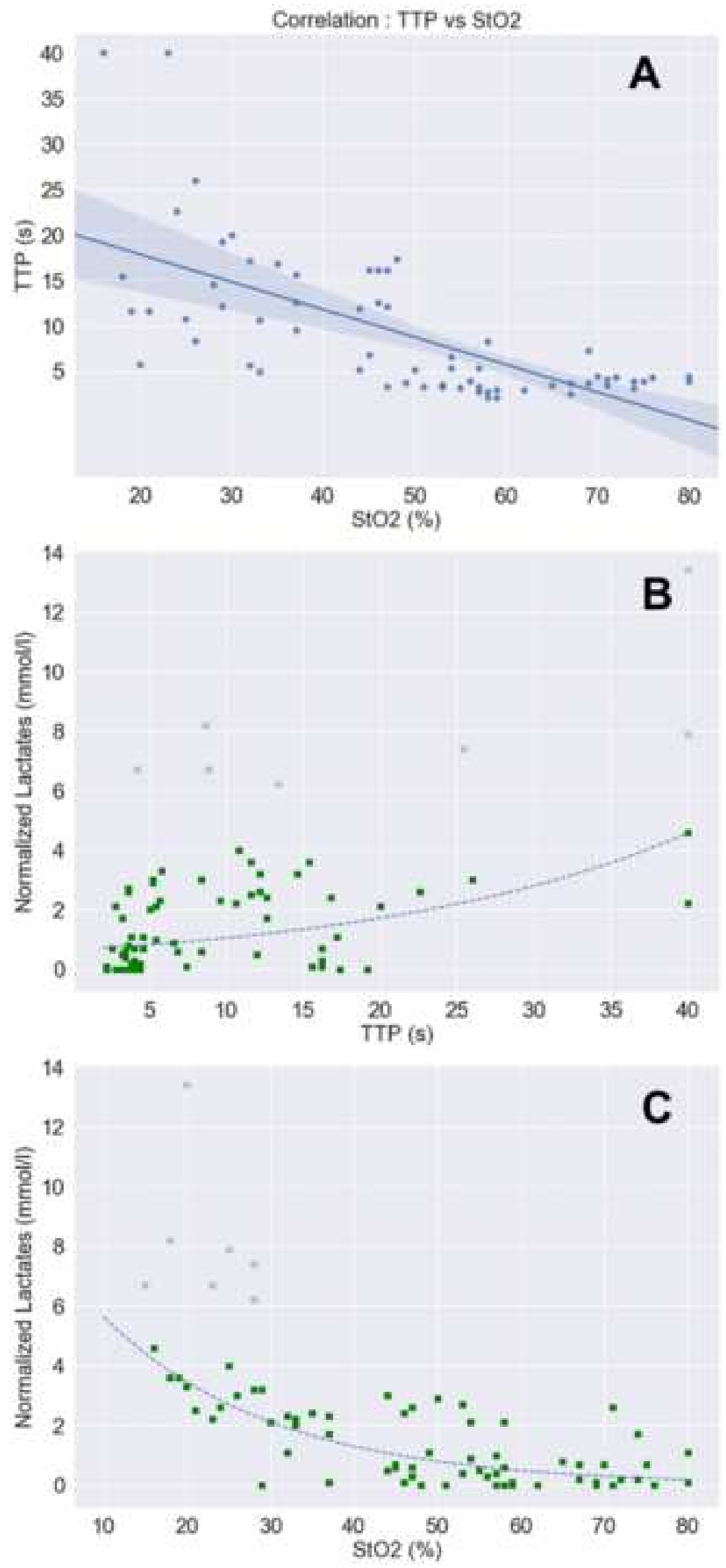


\section{Cumulative error : FLER vs. HYPER}

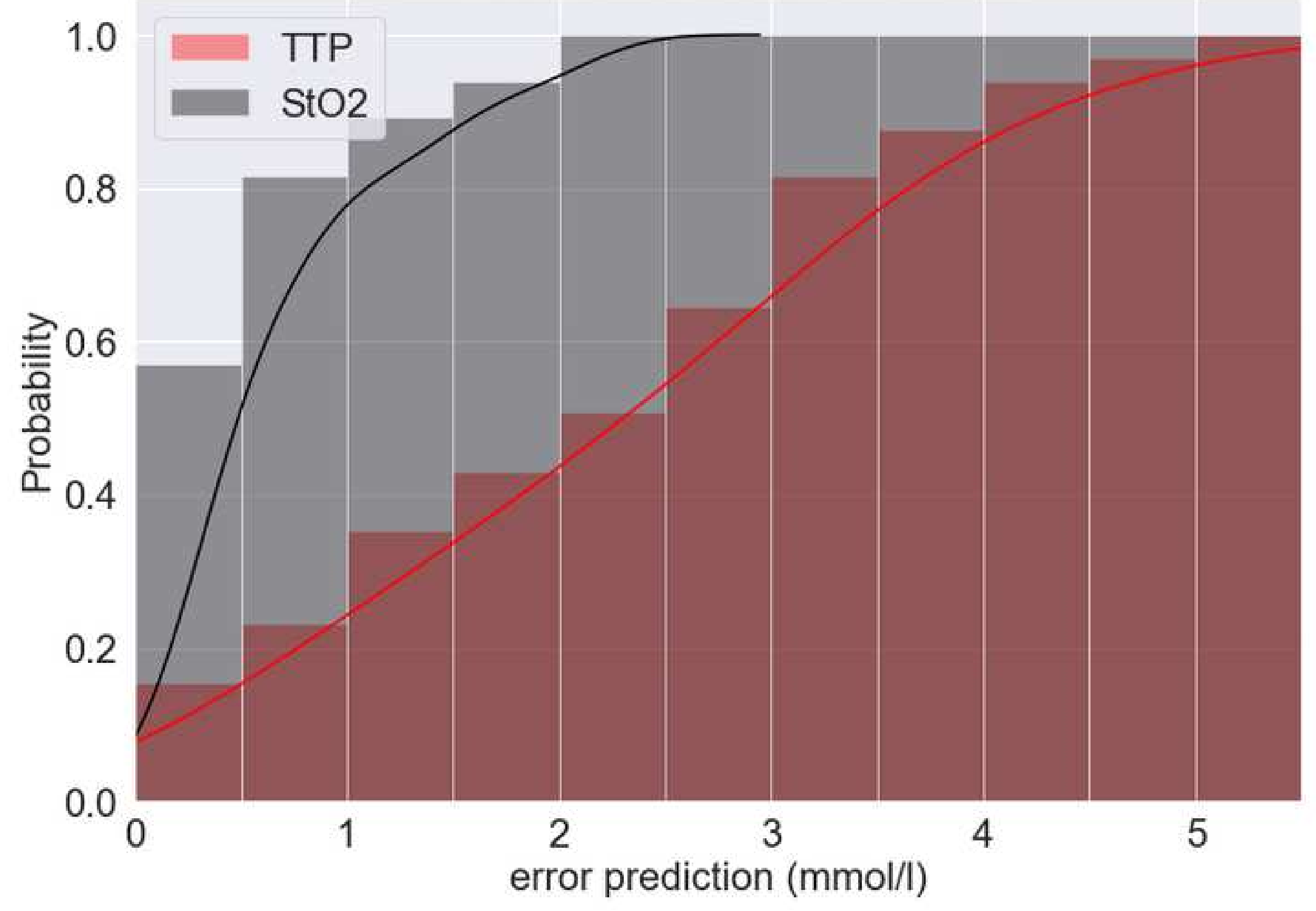




\section{R1 EDITED BY DR SARR}

Quantitative fluorescence angiography vs. hyperspectral imaging to assess bowel ischemia: a comparative study in enhanced reality

Manuel Barberio ${ }^{1,3,4}$, MD; Eric Felli ${ }^{1}$; Emilie Seyller ${ }^{1}$, MD; Fabio Longo ${ }^{1}$, MD; Manish $\mathrm{Chand}^{2}$, MD, PhD; Ines Gockel ${ }^{3}$, MD, MBA; Bernard Geny ${ }^{4}, \mathrm{MD}, \mathrm{PhD}$; Lee Swanström ${ }^{1}, \mathrm{MD}$, PhD; Jacques Marescaux ${ }^{1,5}$, MD, FACS, (Hon) FRCS, (Hon) FJSES, (Hon) FASA; Vincent Agnus $^{1}, \mathrm{PhD}$; Michele Diana ${ }^{1,4,5}, \mathrm{MD}, \mathrm{PhD}$

1) IHU-Strasbourg Institute of Image-Guided Surgery, Strasbourg, France

2) Division of Surgery \& Interventional Science, University College London, London, UK

3) Department of Visceral, Transplant, Thoracic and Vascular Surgery, University Hospital of Leipzig, Leipzig, Germany

4) EA 3072, Fédération de Médecine Translationnelle de Strasbourg, Medical University of Strasbourg, Strasbourg, France

5) Research Institute against Digestive Cancer (IRCAD), Strasbourg, France

\section{Corresponding author:}

Manuel Barberio, MD

IHU-Strasbourg, Institute of Hybrid Image-Guided Surgery, Strasbourg, France 1, place de l'Hôpital, 67091 Strasbourg, France

Email : manuel.barberio@ihu-strasbourg.eu

Phone: +33768281308

A partial overview of this work was presented at the 27th European Association for Endoscopic Surgery (EAES) congress in Seville, Spain, June 12-15, 2019. 


\begin{abstract}

\section{Background}

FLuorescence-based Enhanced Reality (FLER) is a software that providesing quantitative fluorescence angiography (FA), by computing the fluorescence intensity time-to-peak (TTP), after intravenous injection of indocyanine green (ICG). Hyperspectral imaging (HIS) is a HSI is a contrast-free, optical imaging modality; which measures tissue oxygenation $\left(\mathrm{StO}_{2}\right)$.

\section{Methods}

In 8 pigs, an ischemic bowel segment-was created by dividing the arcade branches wsand imaged using $\mathrm{HSI}$ and FLER. $\mathrm{StO}_{2}$ values were acquired through a hyperspectral imaging (HSI) system. Subsequently, fluorescence angiograhyFA was performed using a near-infrared laparoscopic camera, after intravenous injection of $0.2 \mathrm{mg} / \mathrm{kg}$ of ICG. The TTP fluorescence signal was analyzed through a proprietary software to realize a perfusion map. This was overlaid onto real-time images to obtain FLER. Simultaneously, nine adjacent regions of interest (ROIs) were selected and superimposed onto the real-time video, thereby obtaining HYperspectral-based Enhanced Reality (HYPER). FLER and HYPER were superimposed allowing a comparison of both imaging modalities. Local capillary lactate levels s (LCL)-were sampled at the regions of interest ROIs. Two LCL prediction models using the local capillary lactate levels were extrapolated based on both imaging.
\end{abstract}

\title{
Results
}

For all regions of interest, ROIs the mean local capillary lactate levels wereLCL measured $4.67 \pm 4.34 \mathrm{mmol} / \mathrm{L}$, the mean $\mathrm{StO}_{2}$ was $45.92 \pm 18.959 \%$, and the mean TTP was $10.33 \pm 9.436$ sec. Pearson's test between FLER-TTP and HSI-StO2 at the corresponding regions of interest ROIs gave an $\mathrm{R}=-0.66(\mathrm{p}<0.0001)$. The HSI lactate prediction model proved significantly-more accurate than the FLER-based modelene $(\mathrm{p}<0.0001)$.

\section{Conclusion}

Bowel perfusion was elearly quantified using HSI and fluorescence angiograhy FA. HSI yielded more accurate results than fluorescence angiograhy-FA. HYPER may prove to be might become a useful, contrast-free intraoperative tool to quantify bowel ischemia. in a contrast-free fashion. 
1

2

3

4

5

6

7

8

9

10

11

12

13

14

15

16

17

18

19

20

21

22

23

24

25

26

27

28

29

30

31

32

33

34

35

36

37

38

39

40

41

42

43

44

45

46

47

48

49

50

51

52

53

54

55

56

57

58

59

60

61

62

63

64

65

Keywords: fluorescence imaging, quantitative fluorescence angiography, hyperspectral imaging, imaging spectrometer, bowel perfusion, enhanced reality, local capillary lactate. 


\section{Background}

The incidence of gastrointestinal anastomotic leakage_(AL) remains high in certain high risk procedures, ranging from 20 to $35 \%^{1}$ after esophageal procedures, and from 4 to $19 \%^{2}$ after colorectal procedures. In aAdditionally, Aanastomotic leakaget is associated with high morbidity and mortality rates ${ }^{3} . \underline{\text { Inadequate (NO NEW PARAGRAPH) }}$

Inadequate perfusion to the bowel ends being anastomosed remains the most important determinant of anastomotic breakdown ${ }^{3}$. The clinical intraoperative evaluation of gastrointestinal perfusion intraoperatively is unreliable irrespective of the surgeon's experience ${ }^{4}$.

Fluorescence angiography (FA) is a real-time optical imaging technique, which allows the to estimatione of bowel perfusion through the enhanced visualization of an intravenously injected fluorescent dye, most commonly indocyanine green (ICG); using a Near-Infrared (NIR) camera system. Several clinical trials have pointed out that the use of FA intraoperatively holds some potential in preventing anastomotic leak $\mathrm{AL}^{5-9}$, but . However, a standardized protocol for FA and a quantitative metric of the fluorescent signal are currently lckingmissing ${ }^{10}$.

Fluorescence-based Enhanced Reality (FLER) is a software solution; which computes the timeto-peak (TTP) of the fluorescent signal pixel-by-pixel during FA. The accuracy of thise quantification software has been validated in the large animal model using robust biologicat perfusion markers, including local capillary lactate levelss (LCL), measures of mitochondrial respiration $_{2}$ and metabolomics ${ }^{11-14}$.

Hyperspectral imaging (HSI) is another optical imaging technique, which combines a spectroscope and a photo camera, allowing for a contrast-free, real-time, qualitative and quantitative tissue analysis based on tissue oxygen saturation $\left(\mathrm{StO}_{2}\right)^{15}, 16$. The limitation downside-of HSI, at the present stage of development- is in the lack of a video rate and the absence of a minimally invasive device. Through a customization of the HSI system and a 
proprietary software, static HSI perfusion images can be superimposed onto a real-time video, in order to generate an HSI-powered enhanced reality (HYPER) environment, thereby allowing for a precise surgical navigation. The accuracy of HYPER in quantifying fromearly (5 minutes) to late (360 minutes) bowel ischemia using reliable markers of cellular injury cell-suffering surrogates, such as LCL and the generation of reactive oxygen species (ROS) production, has been previously demonstrated previously ${ }^{17}$. The aim of the present study was to compare the accuracy of FLER and HYPER in a porcine, non-survival, open surgery model of bowel ischemia. 


\section{Methods}

Animal characteristics

Eight adult male pigs (Large White, mean weight: $36.6 \pm 6.2 \mathrm{~kg}$ ) were included in the present study, which wais part of the ELIOS project (Endoscopic Luminescent Imaging for Oncology Surgery). This study was; approved by the local Ethical Committee on Animal Experimentation (ICOMETH No. 38.2016.01.085); and by the French Ministry of Superior Education and Research (MESR) (APAFIS\#8721-2017013010316298-v2). Only male pigs weresex was chosen in order to have a homogeneous cohort to studysubjects' sample. All animals were managed according to French laws for animal use and care, and all experiments were performed according to the directives of the European Community Council (2010/63/EU) and ARRIVE guidelines $^{18}$.

The animals were fasted for 24 hours with free access to water before-the experimentsurgery. Premedication was administered 10 minutes before-starting the experimentsurgery, using an intramuscular injection of ketamine $(20 \mathrm{mg} / \mathrm{kg})$ and azaperone $(2 \mathrm{mg} / \mathrm{kg})$ (Stresnil, JanssenCilag, Belgium). Intravenous propofol $(3 \mathrm{mg} / \mathrm{kg})$ combined with rocuronium $(0.8 \mathrm{mg} / \mathrm{kg})$ was used for induction with a. Anesthesia maintained with $2 \%$ isoflurane. At the end of the procedures, pigs were sacrifickilled with an intravenous injection of Pentobarbital Sodium $(40 \mathrm{mg} / \mathrm{kg})\left(\right.$ Exagon ${ }^{\circ}$, AXIENCE, France), while still under 5\% isoflurane anesthetizedsia.

\section{Hyperspectral-based enhanced reality}

We used aA CMOS hyperspectral push-broom imaging system (TIVITA®, Diaspective Vision $\mathrm{GmbH}$, Germany) withwas used; the camera lens was placed at a distance of $35 \mathrm{~cm}$ from the operativesurgical scene. The HSI camera is able to quantify $\mathrm{StO}_{2}$ by generating pseudo-color images as an instantaneous output (acquisition time: roughly $6 \mathrm{sec}$ ), by means of the integrated software. As previously-described previously ${ }^{17}$, the hardware was customized; by adding an aligned video-camera and an infrared distance sensor. A Python ${ }^{\mathrm{TM}}$-based proprietary software 
allowed thee superimpositione the $\mathrm{StO}_{2}$ quantification images onto the real-time video of the operativesurgical field, athereby producing nd HYPER was obtained.

\section{Fluorescence-based Enhanced Reality}

After an intravenous injection of $0.2 \mathrm{mg} / \mathrm{kg}$ Indocyanine Green (ICG) (Infracyanine ${ }^{\circledR}$, Serb, Paris, France), the fluorescent signal was captured with a 30-degree near-infrared laparoscope (D-Light P, KARL STORZ Endoscope, Tuttlingen, Germany), placed at the shortest distance required to capture the entire operative surgical-area of interest. The ER-PERFUSION software (IRCAD, France) computes perfusion as the TTP of the fluorescent signal evolution pixel-bypixel.

TTP is converted into a quantitative perfusion cartography- generated by analyzing the velocity of the fluorescence signal until it reaches its maximal peak of intensity-peak. The perfusion cartography is a color-coded image; which can be used as a last image hold function and overlaid onto the real-time laparoscopic video. Fluorescence intensity varies largely depending on the distance between the NIR camera and the target. BecauseSince-we used the relative fluorescence signal variation over time, our measure was not influenced by distance. TTP is obtained as a difference $T_{75}-T_{25}$, where $T_{25}$ and $T_{75}$ represent time_points corresponding to the first and last quartile of fluorescent intensity over time respectively (Figure 1). The difference between $\mathrm{T}_{100}$ (maximum intensity of fluorescent signal) and $\mathrm{T}_{0}$ (minimum intensity of fluorescent signal), has been used previously used-to compute $\mathrm{TTP}^{19}{ }_{2}=\mathrm{h}$ However, it is often difficult to preciselydetermine precisely the time_points of the minimum (given the frequent presence of signal noise) and maximum (given the presence of a long signal plateau phase) fluorescence intensity. As a result, $\mathrm{T}_{25}$ and $\mathrm{T}_{75}$ were chosen, becausesince they are more robust time_points. 
An ICG reference card (Green Balance ${ }^{\mathrm{TM}}$, Diagnostic Green GmbH, Aschheim-Dornach, Germany) was placed in the operativesurgical-field during FLER acquisitions in order to test the fluorescence signal before ICG injection.

The software generated a quantitative perfusion map, which was superimposed onto the laparoscopic real-time video to obtain FLER, as previously-described previously ${ }^{11-14}$ (Figure 2).

\section{Analysis of Capillary lactate levelsanalysis}

Using the level of local capillary lactates (LCL) as the primary outcome, a sample size calculation was performed based on previous works from our group; using a similar experimental model. Using a superiority design, 50 simultaneously analyzed spots with both techniques were required to have a $90 \%$ chance of detecting, as significant at the $5 \%$ level, a difference in the primary outcome measure at a statistical significance of 5\%. Because Since an ischemic bowel loop was created in each animal and each loop contained 9 regions of interest (ROIs), 8 animals (72 ROIs) in total were included.

LCL served as "ground-truth" metric to compare the performances of FLER and HYPER performances. A strip-based, portable lactate analyzer (EDGE®, ApexBio, Taipei, Taiwan; measuring range: $1.1-22.2 \mathrm{mmol} / \mathrm{L}$ ) was used to assess lactate levels on blood punctured from the serosal capillary vessels in correspondence with each identified ROI. The order of sampling was randomized. The robustness of LCL to quantify the perfusion of the gastrointestinal tract has been validatedlargely previouslydemenstrated in the past ${ }^{11-14,17,20,21}$.

Systemic lactatemia was measured on blood samples obtained by puncturing capillary vessels on the pigs' snout and LCLs were normalized to the systemic values.

\section{OperativeSurgicat-set-up and experimental workflow}


A laparotomy was performed, and a $10 \mathrm{~cm}$ ischemic segment was created in the proximal jejunum, by dividing the arcade branches (Figure 3). After 30 minutes of ischemia, HYPER was first performed, displaying the $\mathrm{StO}_{2}$ pseudo-color images onto the real-time laparoscopic video shown directly on the OR monitor. Nine contiguous regions of interest (ROIs), including perfused and ischemic areas, were placed randomly placed throughout the length of the bowel loop and displayed in augmented reality. Immediately afterwards, ICG was administered intravenously, and FLER was obtained and simultaneously superimposed onto HYPER (Figure 2). As next step, the _sampling of LCLs were obtained in randomized order at the 9 ROIs displayed in augmented reality was performed in randomized order.

The HSI images weare obtained in approximately 6 secends for each acquisition withand the $\mathrm{StO}_{2}$ cartography wais provided instantaneously. The time required to position the ROIs and obtain HYPER wais approximately 1 minute. The delay between ICG administration, in order to perform FA and superimposition of FLER onto HYPER wais approximately of 2 minttes. Therefore, the two imaging techniques were performed with a time interval of 1 mintte. The delay between HYPER completion and lactate sampling was of roughly 3 minutes.

In order to minimize any bias; possibly generated by motion or light artefacts, breathing was stopped, and external light interference was prevented during the time required to obtain both imaging acquisitions ( 6 secends for HSI and 40 secends for FA).

\section{Prediction of local capillary lactates from FLER TTP and HSI StO2}

The relationship between LCLs and FLER TTP and $\mathrm{HSI} \mathrm{StO}_{2}$ respectively, followed an exponential trend, which was modeled by an exponential regression analysis. This technique allowed us to generate a prediction algorithm of the LCL values from the corresponding FLER TTP and HSI-StO ${ }_{2}$. The following fitting functions were found:

1) based on the FLER TTP: 
predicted $L C L=e^{0.01 * T T P-0.553}+$ SystemicLactates

2) based on $\mathrm{HSI}-\mathrm{StO}_{2}$ :

predictedLCL $=e^{-0.049 * \mathrm{StO}_{2}+2.22}+$ SystemicLactates

In order to validate the prediction models $s_{2}$ a mean square minimization of the objective function $\exp (-a x+b)$ was performed on normalized LCLlactates; using the Scipy, Python library ${ }^{22}$. The prediction functions were obtained from the whole datasets, becausesince no significancetive difference in terms of error prediction could be observed when performing a leave-one-(pig) out cross-validation.

The accuracy of both models, indicated as the absolute difference between the sampled LCLs values and the predicted LCL values of both prediction models, was computed on the whole data by the corresponding exponential equation.

\section{Statistical analysis}

Data are shown as mean \pm SD unless indicated otherwise. Statistics were performed using the Python Scikit-learn library ${ }^{23}$. A Pearson's test was used to compare variables showing a linear relationship. Spearman correlation and exponential regression were used to investigate variables presenting a non-linear relationship. A Wilcoxon test was performed for paired comparison of the lactate prediction algorithms (based on HYPER and on FLER), sincebecause a non-Gaussian data distribution was observed. A $p$ value $<0.05$ was considered statistically significant. 


\section{Results}

Using tThe enhanced reality, we were able allowed to precisely-identify precisely the ROIs on the bowel loops $(\mathrm{n}=72)$ as demonstrated by both HSI-StO2 and FLER TTP, thereby allowing enabling accurate sampling of serosal capillary blood to measure LCL. Mean systemic lactate concentration was $2.63 \pm 2.85 \mathrm{mmol} / \mathrm{L}$. Considering all the ROIs, the mean LCL concentration was $4.67 \pm 4.34 \mathrm{mmol} / \mathrm{L}$, the mean normalized $\mathrm{LCL}$ value was $2.034 \pm 2.44 \mathrm{mmol} / \mathrm{L}$, the mean $\mathrm{StO}_{2}$ value was $45.92 \pm 18.659 \%$ and the mean TTP was $10.33 \pm 9.436$ seconds.

Correlation between FLER TTP and HSI-StO2

The Pearson analysis between FLER TTP and HSI-StO2 in correspondence with the same ROIs gave a $\mathrm{R}=-0.66(\mathrm{p}<0.0001)($ Figure $4 \mathbf{a})$.

Correlation between LCL and TTP / LCL and StO2

The Spearman correlation between LCL salues and FLER TTP gave a $\mathrm{R}=0.40(\mathrm{p}=0.001)$, and a $\mathrm{R}=-0.62(\mathrm{p}<0.0001)$ between LCL-values and HSI-StO2. Both correlations produced 7 outliers (Figure 4 b-c), including only completely ischemic ROIs, with LCL > $6 \mathrm{mmol} / \mathrm{l}$. These 7 outliers were excluded from the analysis of the accuracy of the LCL prediction models accuracy reported below.

LCL prediction based on FLER TTP

The mean error of the lactate prediction model was $0.95 \pm 0.74 \mathrm{mmol} / \mathrm{L}$. The median error was $0.68 \mathrm{mmol} / \mathrm{L}$, and $=95 \%$ of the errors occurred for LCLstactate values $<2.33 \mathrm{mmol} / \mathrm{L}$. LCL prediction based on $\mathrm{HSI}-\mathrm{StO} 2$

The lactate prediction model showed a mean error of $0.65 \pm 0.59 \mathrm{mmol} / \mathrm{L}$, with a median error of $0.43 \mathrm{mmol} / \mathrm{L}_{2}=95 \%$ of the errors occurred for LCLstactate values $<2 \mathrm{mmol} / \mathrm{L}$.

Comparison between FLER-based and HSI-based lactate prediction models 
1

2

3

4 ( $\mathrm{p}<0.0001$ ), with HSI proviyielding a better lactate prediction performance (Figure 5). 


\section{Discussion}

The results of the present study show that both FLER and HYPER give virtually real-time information, and the metrics used by both quantitative methods, namely TTP and $\mathrm{StO}_{2}$, are significantly correlate welle. Additionally, both parameters have a statistically significant exponential relationship with the- LCLseapillary lactates. A fitting function between LCL and both the FLER TTP and HSI-StO 2 was found and provided two LCL prediction models. It was observed that $\underline{B}$ both prediction models were less accurate for LCL-values $>6 \mathrm{mmol} / \mathrm{L}$. As a result, a threshold below this value was set which, and led to excluding a total of 7 ROIs which were definitely outliers (Figure 4 b-c). This correction lead to a substantially improved precision of the prediction algorithms. Theseis findings are is in line with the results of our prior study, in which HYPER provided a significantly better accuracy of the LCL prediction for $\mathrm{StO}_{2}$ values $>30 \%{ }^{17}$. In the present study, all outlying ROIs (LCL $>6 \mathrm{mmol} / \mathrm{L}$ ) showed that $\mathrm{StO}_{2}$ was $<30 \%$. Likewise, it is clinically irrelevant to precisely-predict the LCL in ROIs precisely which are frankly ischemic based on a quantitative optical imaging analysis and which are often identifiable as non-perfused areas to the naked eye. Both prediction models showed high accuracy in discriminating marginally perfused areas, which are often generally difficult to identify locate under white light. The precise identification of those areas is important not only to assess the perfusion of gastrointestinal anastomoses, but also to evaluate the bowel viability after mesenteric ischemia. As outlined by Kougias et al., second-look laparotomies with additional bowel resections are required in $57 \%$ of cases presenting with mesenteric ischemia ${ }^{24}$. Therefore, non-invasive optical imaging tools, like FLER or HYPER, could improve the intraoperative decision-making process.-We must stress, however, that However, the threshold of those imaging modalities representing the "point of no return", irreversible ischemia, of bowel viability has yet to be identified and is currently under investigation. 
The prediction model based on the FLER TTP was significantly-less accurate when compared to the model based on the $\mathrm{HSI} \mathrm{StO}_{2}$-based model. A possible explanation could be the short ischemia period (30 minutes) chosen in the present set-up. During this time, macroscopic signs of tissue injury suffering are rarely found. However, there are, however, intracellular changes which decreaseprovoke a reduced-mitochondrial activity. As a result, decreases in reduced- $\mathrm{O}_{2}$ and increases $\underline{\text { in LCL production already occur }}{ }^{14}$. The HSI-detected $\mathrm{StO}_{2}$ seems to better mirror those intracellular metabolic changes as compared to FLER, which finally measures a macroscopic phenomenon such as the serosal surface blood flow.

FA is a well-established intraoperative method to assess gastrointestinal perfusion and is relatively easy to integrate in the surgical workflow ${ }^{9}$, but . However, there is still no consensus on the impact of FA on reducdecreasing the incidence of anastomotic leakage, $\mathrm{AL}$ and the results of randomized clinical trials are awaited ${ }^{25}$. Two factors affect the performances of FA and should be controlled. The first factorene is the progressive diffusion over time of the fluorophore through the serosal capillary network into the margins of the ischemic zones-over time, leading to an overestimation of the vascularized area. The second factorene is the quadratic inverse relationship between the target-endoscope distance and fluorescence intensity ${ }^{11,14}$. When distance is not controlled and/or fluorescence intensity is not normalized with a calibration system, the presence of a fluorescent signal in the tissue is not representative of the true perfusion. To overcome such interpretation biases, a software-based quantitative FA was introduced- based on the analysis of the dynamics of the fluorescent signal-dynamies 11,14 , 19. Wada et al. successfully created an algorithm of quantitative fluorescence analysis during clinical colorectal cases ${ }^{19}$. The authors used a similar algorithm of evaluating the fluorescence dynamics evaluation ${ }^{11}$; hHowever, unlike FLER, the enhanced information (virtual perfusion cartography) was not superimposed onto real-time images. FLER is also currently being evaluated in a clinical trial ${ }^{26},{ }^{27}$ (https://clinicaltrials.gov/ct2/show/NCT02626091) with 
promising early results in terms of correlation with surrogate markers of perfusion and prediction of anastomotic complications.

HSI has been used for decades in various industrial sectors or for remote sensing ${ }^{28}$. Recently, HSI was used successfully used-in medical and surgical applications, including identification of various tumors identifieation ${ }^{28}$, intraoperative parathyroid recognition ${ }^{29}$, and real-time quantitative assessment of perfusion assessment during gastrointestinal operationssurgical procedures ${ }^{30-32}$.

Compared to fluorescence imaging, HSI provides a greatlarger amount of information and is able to quantify several tissue components, including the content of water, oxygen, and hemoglobin-entent. Additionally, a remarkable advantage when compared to FA is that HSI does not require the use of any contrast agents, thus, - In other words, - HSI is an optical, contrast-free, and non-destructive in vivo "biopsy" of the operative surgical-field, allowing one to virtually discriminate anatomicat structures and characterize certain aspects of tissue physiology. However, $t$ The large HSI-generated datasets, however, require complex data processing algorithms in order to extrapolate useful discriminative features from the spectral curves. The TIVITA ${ }^{\circledR}$ HSI system has a built-in software; which generates pseudo-color images by, quantifying $\mathrm{StO}_{2}{ }^{29-31}$. We used a proprietary software solution for the superimposing of the $\mathrm{HSI} \mathrm{StO}_{2}$ images onto real-time video images in order to create an enhanced reality surgical scene (HYPER) and to preciselyidentify precisely the small ROIs on the bowel surface.

To the best of our knowledge, the present study is the first comparison between HSI and

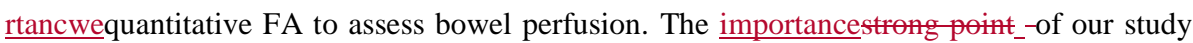
lies in its robust design and methodology. Limitations of this study lie in the fact that the results are obtained in controlled experimental conditions of early ischemia (30 minutes) and the clinical application of HYPER has not been demonstrated yet. Furthermore, although the 
acquisitions of the two imaging systems occurred virtually simultaneously (1-minute delay), the study draws on comparison between a minimally invasive NIR camera and an open-surgery HSI camera. In this setting, HYPER displayed a signifieantly-better overall accuracy. While this difference was statistically significant, it remains unknown if tjis diofference it willeuld prove to be clinically importantsignificant.

Currently, However, at the moment, HSI systems are limited by the lack of an adequately fast video rate and by the lack of a system for minimally invasive surgery-system. Some authors have proposed endoscopic HSI systems ${ }^{33,34}$. but- However, the spatial resolution and speed of the data processing speed-are still limited enough to allow its use in our preventdaily surgical practice-implementation.

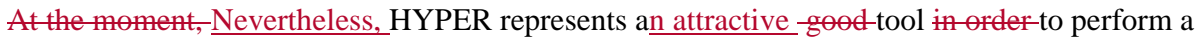
non-invasive, contrast-free, real-time and accurate, intraoperative perfusion quantification.

As next step, a trial to assess the accuracy of HYPER intraoperatively is currently being designed.

\section{Conclusions}

In the acute experimental setting, quantitative evaluation of perfusion using hyperspectral imaging was more accurate when compared to quantitative fluorescence-based evaluation. The next step is clinical transtation and the evaluation of the impact of those emerging technologies on the incidence of anastomotic leakage.

\section{Acknowledgments}

Authors are grateful to Guy Temporal, Christopher Burel, and Camille Goustiaux, professionals in medical English proofreading, for their valuable help in revising the manuscript. 
COI/DISCLOSURES: Jacques Marescaux is the President of both IRCAD and IHU Strasbourg Institutes, which are partly funded by KARL STORZ, Siemens, and Medtronic. Lee Swanström is the Scientific Director of the IHU and Michele Diana is the recipient of the ELIOS grant. Manish Chand has received fees from Stryker Endoscopy for his work as a consultant for fluorescent angiography, outside of this work. The remaining authors have no conflicts of interest or financial ties to disclose.

FUNDING/SUPPORT-source: This work was funded by the ARC Foundation through the $\underline{\text { ELIOS (Endoscopic Luminescent Imaging for precision Oncologic Surgery) grant }}$

This work was funded by the ARC Foundation through the ELIOS (Endoscopic Luminescent Imaging for precision Oncologic Surgery) grant.

Formatted: Space Before: Auto, After: Auto, Line spacing: single

\section{References:}

1. Campbell C, Reames MK, Robinson M, Symanowski J, Salo JC. Conduit Vascular Evaluation is Associated with Reduction in Anastomotic Leak After Esophagectomy. J Gastrointest Surg. 2015;19:806-12.

2. Karliczek A, Benaron DA, Baas PC, Zeebregts CJ, Wiggers T, van Dam GM. Intraoperative assessment of microperfusion with visible light spectroscopy for prediction of anastomotic leakage in colorectal anastomoses. Colorectal Dis.12:1018-25.

3. Urbanavicius L, Pattyn P, de Putte DV, Venskutonis D. How to assess intestinal viability during surgery: A review of techniques. World J Gastrointest Surg. 2011;3:59-69.

4. Karliczek A, Harlaar NJ, Zeebregts CJ, Wiggers T, Baas PC, van Dam GM. Surgeons lack predictive accuracy for anastomotic leakage in gastrointestinal surgery. Int J Colorectal Dis. 2009;24:569-76.

5. Kudszus S, Roesel C, Schachtrupp A, Hoer JJ. Intraoperative laser fluorescence angiography in colorectal surgery: a noninvasive analysis to reduce the rate of anastomotic leakage. Langenbecks Arch Surg. 2010;395:1025-30.

6. Jafari MD, Wexner SD, Martz JE, McLemore EC, Margolin DA, Sherwinter DA, et al. Perfusion assessment in laparoscopic left-sided/anterior resection (PILLAR II): a multiinstitutional study. J Am Coll Surg. 2015;220:82-92 e1.

7. Ris F, Liot E, Buchs NC, Kraus R, Ismael G, Belfontali V, et al. Multicentre phase II trial of near-infrared imaging in elective colorectal surgery. Br J Surg. 2018;105:1359-67.

8. De Nardi P, Elmore U, Maggi G, Maggiore R, Boni L, Cassinotti E, et al. Intraoperative angiography with indocyanine green to assess anastomosis perfusion in patients 
undergoing laparoscopic colorectal resection: results of a multicenter randomized controlled trial. Surg Endosc. 2019.

9. Blanco-Colino R, Espin-Basany E. Intraoperative use of ICG fluorescence imaging to reduce the risk of anastomotic leakage in colorectal surgery: a systematic review and meta-analysis. Tech Coloproctol. 2018;22:15-23.

10. Vallance A, Wexner S, Berho M, Cahill R, Coleman M, Haboubi N, et al. A collaborative review of the current concepts and challenges of anastomotic leaks in colorectal surgery. Colorectal Dis. 2017;19:01-012.

11. Diana M, Agnus V, Halvax P, Liu YY, Dallemagne B, Schlagowski Al, et al. Intraoperative fluorescence-based enhanced reality laparoscopic real-time imaging to assess bowel perfusion at the anastomotic site in an experimental model. Br J Surg. 2015;102:e16976.

12. Diana M, Dallemagne B, Chung H, Nagao Y, Halvax P, Agnus V, et al. Probe-based confocal laser endomicroscopy and fluorescence-based enhanced reality for real-time assessment of intestinal microcirculation in a porcine model of sigmoid ischemia. Surg Endosc. 2014;28:3224-33.

13. Diana M, Halvax P, Dallemagne B, Nagao Y, Diemunsch P, Charles AL, et al. Real-time navigation by fluorescence-based enhanced reality for precise estimation of future anastomotic site in digestive surgery. Surg Endosc. 2014;28:3108-18.

14. Diana M, Noll E, Diemunsch P, Dallemagne B, Benahmed MA, Agnus V, et al. Enhancedreality video fluorescence: a real-time assessment of intestinal viability. Ann Surg. 2014;259:700-7.

15. Grambow E, Dau M, Holmer A, Lipp V, Frerich B, Klar E, et al. Hyperspectral imaging for monitoring of perfusion failure upon microvascular anastomosis in the rat hind limb. Microvasc Res. 2018;116:64-70.

16. Holmer A, Marotz J, Wahl P, Dau M, Kammerer PW. Hyperspectral imaging in perfusion and wound diagnostics - methods and algorithms for the determination of tissue parameters. Biomed Tech (Berl). 2018;63:547-56.

17. Barberio M, Longo F, Fiorillo C, Seeliger B, Mascagni P, Agnus V, et al. HYPerspectral Enhanced Reality (HYPER): a physiology-based surgical guidance tool. Surg Endosc. 2019.

18. Kilkenny C, Browne W, Cuthill IC, Emerson M, Altman DG, Group NCRRGW. Animal research: reporting in vivo experiments: the ARRIVE guidelines. J Gene Med. 2010;12:561-3.

19. Wada T, Kawada K, Takahashi R, Yoshitomi M, Hida K, Hasegawa S, et al. ICG fluorescence imaging for quantitative evaluation of colonic perfusion in laparoscopic colorectal surgery. Surg Endosc. 2017;31:4184-93.

20. Diana M, Noll E, Charles AL, Diemunsch P, Geny B, Liu YY, et al. Precision real-time evaluation of bowel perfusion: accuracy of confocal endomicroscopy assessment of stoma in a controlled hemorrhagic shock model. Surg Endosc. 2017;31:680-91.

21. Diana M, Noll E, Diemunsch P, Moussallieh FM, Namer IJ, Charles AL, et al. MetabolismGuided Bowel Resection: Potential Role and Accuracy of Instant Capillary Lactates to Identify the Optimal Resection Site. Surg Innov. 2015;22:453-61.

22. Virtanen P, Gommers R, Oliphant TE, Haberland M, Reddy T, Cournapeau D, et al. SciPy 1.0--Fundamental Algorithms for Scientific Computing in Python. arXiv preprint arXiv:190710121. 2019. 
23. Pedregosa F, Varoquaux G, Gramfort A, Michel V, Thirion B, Grisel O, et al. Scikit-learn: Machine learning in Python. Journal of machine learning research. 2011;12:2825-30.

24. Kougias P, Lau D, El Sayed HF, Zhou W, Huynh TT, Lin PH. Determinants of mortality and treatment outcome following surgical interventions for acute mesenteric ischemia. Journal of vascular surgery. 2007;46:467-74.

25. Armstrong G, Croft J, Corrigan N, Brown JM, Goh V, Quirke P, et al. IntAct: intraoperative fluorescence angiography to prevent anastomotic leak in rectal cancer surgery: a randomized controlled trial. Colorectal Dis. 2018;20:0226-034.

26. Seeliger B, Barberio M, D'Urso A, Agnus V, Longo F, Mascagni $P$, et al. Fluorescence in rectal cancer surgery. Annals of Laparoscopic and Endoscopic Surgery. 2018;3:47

27. Mascagni P, Longo F, Barberio M, Seeliger B, Agnus V, Saccomandi P, et al. New intraoperative imaging technologies: Innovating the surgeon's eye toward surgical precision. J Surg Oncol. 2018;118:265-82.

28. Lu G, Fei B. Medical hyperspectral imaging: a review. J Biomed Opt. 2014;19:10901.

29. Barberio M, Maktabi M, Gockel I, Rayes N, Jansen-Winkeln B, Köhler $H$, et al. Hyperspectral based discrimination of thyroid and parathyroid during surgery. Current Directions in Biomedical Engineering2018. p. 399.

30. Jansen-Winkeln B, Maktabi M, Takoh JP, Rabe SM, Barberio $M$, Kohler $\mathrm{H}$, et al. [Hyperspectral imaging of gastrointestinal anastomoses]. Chirurg. 2018;89:717-25.

31. Kohler H, Jansen-Winkeln B, Maktabi M, Barberio M, Takoh J, Holfert N, et al. Evaluation of hyperspectral imaging (HSI) for the measurement of ischemic conditioning effects of the gastric conduit during esophagectomy. Surg Endosc. 2019.

32. Jansen-Winkeln B, Holfert N, Kohler H, Moulla $Y$, Takoh JP, Rabe SM, et al. Determination of the transection margin during colorectal resection with hyperspectral imaging (HSI). Int J Colorectal Dis. 2019;34:731-9.

33. Clancy NT, Arya S, Stoyanov D, Singh M, Hanna GB, Elson DS. Intraoperative measurement of bowel oxygen saturation using a multispectral imaging laparoscope. Biomed Opt Express. 2015;6:4179-90.

34. Zuzak KJ, Naik SC, Alexandrakis G, Hawkins D, Behbehani K, Livingston E. Intraoperative bile duct visualization using near-infrared hyperspectral video imaging. Am J Surg. 2008;195:491-7.

\section{Figures Legends}

Fig.1: TTP-representation. Graphicat representation of the time-to-peak in at well--perfused (blue) and in an ischemic area (green) areas, in which very different maximum fluorescent intensity peaks (Fmax) weare reached. TTP is the time required (in seconds) for the intensity curve to go from the first to the last quartile of fluorescent intensity over time. As represented, TTP is lesssherter for the perfused zone when compared to the ischemic-zoneene. 
Fig.2: Enhanced reality modalities overlays. Ischemic small bowel model displayed under white light (A), with ICG reference card visible in the center of the bowel loop. FLER (B) with superimposing of the fluorescence-based quantitative perfusion cartography. HYPER (C) generated through the superimposing of the $\mathrm{StO}_{2}$ pseudo-color image captured with HSI. (D) shows the warping of FLER and HYPER simultaneously, over the real-time video captured with the NIR-laparoscopic camera during the white light mode. The selected ROIs are precisely identifiable in the oerativesurgical scene and LCLs were sampled in correspondence with them.

Figure 3: OperativeSurgical set-up. The ischemic loop is placed on top of the calibration chessboard in order to facilitate the accurate superimposing of images obtained from cameras with different angles. The laparoscopic camera (arrow) and the HSI system (arrowhead) are placed in order to capture theoperative-surgieal scene from different angles but with the same picture orientation.

Figure 4: TTP/StO 2 correlation and LCL regression analysis. (A) Visual representation of Pearson's correlation between HSI-StO2 and FLER TTP. The exponential regression correlation between LCLslactates-and both FLER TTP (B) and HSI-StO2 (C) is shown. Seven datasets of highly ischemic regions (shadowed points) were excluded from the regression analysis, becausesince it was observed that, using both imaging modalities, the LCL prediction models weare less accurate when the $\mathrm{LCL}$ was $>6 \mathrm{mmol} / \mathrm{L}$, as highlighted in the graph.

Figure 5: Cumulative error of lactate prediction. Overall precision in predicting LCLlactate_swas-_isttistically significantly highgreater when using the HSI-StO2 (black line) when compared to FLER TTP (red line). 
Quantitative fluorescence angiography vs. hyperspectral imaging to assess bowel ischemia: a comparative study in enhanced reality

Manuel Barberio ${ }^{1,3,4}$, MD; Eric Felli ${ }^{1}$; Emilie Seyller ${ }^{1}$, MD; Fabio Longo ${ }^{1}$, MD; Manish Chand $^{2}$, MD, PhD; Ines Gockel ${ }^{3}$, MD, MBA; Bernard Geny ${ }^{4}$, MD, PhD; Lee Swanström ${ }^{1}, \mathrm{MD}$, PhD; Jacques Marescaux ${ }^{1,5}$, MD, FACS, (Hon) FRCS, (Hon) FJSES, (Hon) FASA; Vincent Agnus $^{1}, \mathrm{PhD}$; Michele Diana ${ }^{1,4}, \mathrm{MD}, \mathrm{PhD}$

1) IHU-Strasbourg Institute of Image-Guided Surgery, Strasbourg, France

2) Division of Surgery \& Interventional Science, University College London, London, UK

3) Department of Visceral, Transplant, Thoracic and Vascular Surgery, University Hospital of Leipzig, Leipzig, Germany

4) EA 3072, Fédération de Médecine Translationnelle de Strasbourg, Medical University of Strasbourg, Strasbourg, France

5) Research Institute against Digestive Cancer (IRCAD), Strasbourg, France

\section{Corresponding author:}

Manuel Barberio, MD

IHU-Strasbourg, Institute of Hybrid Image-Guided Surgery, Strasbourg, France

1, place de l'Hôpital, 67091 Strasbourg, France

Email : manuel.barberio@ihu-strasbourg.eu

Phone: +33768281308

A partial overview of this work was presented at the 27th European Association for Endoscopic Surgery (EAES) congress in Seville, Spain, June 12-15, 2019. 


\begin{abstract}

\section{Background}

FLuorescence-based Enhanced Reality (FLER) is a software that provides quantitative fluorescence angiography by computing the fluorescence intensity time-to-peak (TTP), after intravenous indocyanine green (ICG). Hyperspectral imaging (HIS) is a contrast-free, optical imaging modality which measures tissue oxygenation $\left(\mathrm{StO}_{2}\right)$.
\end{abstract}

\title{
Methods
}

In 8 pigs, an ischemic bowel segment created by dividing the arcade branches ws imaged using HSI and FLER. $\mathrm{StO}_{2}$ values were acquired through a hyperspectral imaging system. Subsequently, fluorescence angiograhy was performed using a near-infrared laparoscopic camera after intravenous injection of $0.2 \mathrm{mg} / \mathrm{kg}$ of ICG. The TTP fluorescence signal was analyzed through a proprietary software to realize a perfusion map. This was overlaid onto realtime images to obtain FLER. Simultaneously, nine adjacent regions of interest were selected and superimposed onto the real-time video, thereby obtaining HYperspectral-based Enhanced Reality (HYPER). FLER and HYPER were superimposed allowing a comparison of both imaging modalities. Local capillary lactate levels were sampled at the regions of interest. Two LCL prediction models using the local capillary lactate levels were extrapolated based on both imaging.

\section{Results}

For all regions of interest, the mean local capillary lactate levels were $4.67 \pm 4.34 \mathrm{mmol} / \mathrm{L}$, the mean $\mathrm{StO}_{2}$ was $45.9 \pm 18.9 \%$, and the mean TTP was 10. $\pm 9.4 \mathrm{sec}$. Pearson's test between FLERTTP and HSI-StO2 at the corresponding regions of interest gave an $\mathrm{R}=-0.66(\mathrm{p}<0.0001)$. The HSI lactate prediction model proved more accurate than the FLER-based model $(p<0.0001)$.

\section{Conclusion}

Bowel perfusion was quantified using HSI and fluorescence angiograhy. HSI yielded more accurate results than fluorescence angiograhy. HYPER may prove to be a useful, contrast-free intraoperative tool to quantify bowel ischemia.

Keywords: fluorescence imaging, quantitative fluorescence angiography, hyperspectral imaging, imaging spectrometer, bowel perfusion, enhanced reality, local capillary lactate. 


\section{Background}

The incidence of gastrointestinal anastomotic leakage remains high in certain high risk procedures, ranging from 20 to $35 \%^{1}$ after esophageal procedures and from 4 to $19 \%^{2}$ after colorectal procedures. In addition Aanastomotic leakage is associated with high morbidity and mortality rates $^{3}$. Inadequate (NO NEW PARAGRAPH)perfusion to the bowel ends being anastomosed remains the most important determinant of anastomotic breakdown ${ }^{3}$. The clinical evaluation of gastrointestinal perfusion intraoperatively is unreliable irrespective of the surgeon's experience ${ }^{4}$.

Fluorescence angiography (FA) is a real-time optical imaging technique which allows the estimation of bowel perfusion through the enhanced visualization of an intravenously injected fluorescent dye, most commonly indocyanine green (ICG); using a Near-Infrared (NIR) camera system. Several clinical trials have pointed out that the use of FA intraoperatively holds some potential in preventing anastomotic leakAL $\mathrm{AL}^{5-9}$, but , a standardized protocol for FA and a quantitative metric of the fluorescent signal are currently lcking ${ }^{10}$.

Fluorescence-based Enhanced Reality (FLER) is a software solution which computes the timeto-peak (TTP) of the fluorescent signal pixel-by-pixel during FA. The accuracy of this quantification software has been validated in the large animal model using robust biologic perfusion markers, including local capillary lactate levels (LCL), measures of mitochondrial respiration, and metabolomics ${ }^{11-14}$.

Hyperspectral imaging (HSI) is another optical imaging technique which combines a spectroscope and a photo camera, allowing for a contrast-free, real-time, qualitative and quantitative tissue analysis based on tissue oxygen saturation $\left(\mathrm{StO}_{2}\right)^{15,16}$. The limitation of HSI at the present stage of development is in the lack of a video rate and the absence of a minimally invasive device. Through a customization of the HSI system and proprietary software, static HSI perfusion images can be superimposed onto a real-time video, in order to generate an HSI- 
powered enhanced reality (HYPER) environment, thereby allowing a precise surgical navigation. The accuracy of HYPER in quantifying early (5 minutes) to late (360 minutes) bowel ischemia using reliable markers of cellular injury, such as LCL and the generation of reactive oxygen species (ROS), has been demonstrated previously ${ }^{17}$. The aim of the present study was to compare the accuracy of FLER and HYPER in a porcine, non-survival, open surgery model of bowel ischemia. 


\section{Methods}

Animal characteristics

Eight adult male pigs (Large White, mean weight: $36.6 \pm 6.2 \mathrm{~kg}$ ) were included in the present study, which was part of the ELIOS project (Endoscopic Luminescent Imaging for Oncology Surgery). This study was approved by the local Ethical Committee on Animal Experimentation (ICOMETH No. 38.2016.01.085) and by the French Ministry of Superior Education and Research (MESR) (APAFIS\#8721-2017013010316298-v2). Only male pigs were chosen in order to have a homogeneous cohort to study. All animals were managed according to French laws for animal use and care, and all experiments were performed according to the directives of the European Community Council (2010/63/EU) and ARRIVE guidelines ${ }^{18}$.

The animals were fasted for $24 \mathrm{~h}$ with free access to water before the experiment. Premedication was administered 10 mins before starting the experiment, using an intramuscular injection of ketamine $(20 \mathrm{mg} / \mathrm{kg})$ and azaperone $(2 \mathrm{mg} / \mathrm{kg})$ (Stresnil, Janssen-Cilag, Belgium). Intravenous propofol $(3 \mathrm{mg} / \mathrm{kg})$ combined with rocuronium $(0.8 \mathrm{mg} / \mathrm{kg})$ was used for induction with anesthesia maintained with $2 \%$ isoflurane. At the end of the procedures, pigs were killed with intravenous Pentobarbital Sodium (40mg/kg) (Exagon®, AXIENCE, France), while still anesthetized.

\section{Hyperspectral-based enhanced reality}

We used a CMOS hyperspectral push-broom imaging system (TIVITA®, Diaspective Vision $\mathrm{GmbH}$, Germany) with the camera lens placed at a distance of $35 \mathrm{~cm}$ from the operative scene. The HSI camera is able to quantify $\mathrm{StO}_{2}$ by generating pseudo-color images as an instantaneous output (acquisition time: roughly $6 \mathrm{~s}$ ) by means of the integrated software. As described previously ${ }^{17}$, the hardware was customized by adding an aligned video-camera and an infrared distance sensor. A Python ${ }^{\mathrm{TM}}$-based proprietary software allowed the superimposition the $\mathrm{StO}_{2}$ 
quantification images onto the real-time video of the operative field, thereby producing

\section{HYPER.}

\section{Fluorescence-based Enhanced Reality}

After an intravenous injection of $0.2 \mathrm{mg} / \mathrm{kg}$ Indocyanine Green (ICG) (Infracyanine ${ }^{\circledR}$, Serb, Paris, France), the fluorescent signal was captured with a 30-degree near-infrared laparoscope (D-Light P, KARL STORZ Endoscope, Tuttlingen, Germany) placed at the shortest distance required to capture the entire operative area of interest. The ER-PERFUSION software (IRCAD, France) computes perfusion as the TTP of the fluorescent signal evolution pixel-bypixel.

TTP is converted into a quantitative perfusion cartography generated by analyzing the velocity of the fluorescence signal until it reaches its maximal peak of intensity. The perfusion cartography is a color-coded image which can be used as a last image hold function and overlaid onto the real-time laparoscopic video. Fluorescence intensity varies largely depending on the distance between the NIR camera and the target. Because we used the relative fluorescence signal variation over time, our measure was not influenced by distance. TTP is obtained as a difference $\mathrm{T}_{75}-\mathrm{T}_{25}$, where $\mathrm{T}_{25}$ and $\mathrm{T}_{75}$ represent time points corresponding to the first and last quartile of fluorescent intensity over time respectively (Figure 1). The difference between $\mathrm{T}_{100}$ (maximum intensity of fluorescent signal) and $\mathrm{T}_{0}$ (minimum intensity of fluorescent signal), has been used previously to compute $\mathrm{TTP}^{19}$; however, it is often difficult to determine precisely the time points of the minimum (given the frequent presence of signal noise) and maximum (given the presence of a long signal plateau phase) fluorescence intensity. As a result, $\mathrm{T}_{25}$ and $\mathrm{T}_{75}$ were chosen, because they are more robust time points.

An ICG reference card (Green Balance ${ }^{\mathrm{TM}}$, Diagnostic Green GmbH, Aschheim-Dornach, Germany) was placed in the operative field during FLER acquisitions in order to test the fluorescence signal before ICG injection. 
The software generated a quantitative perfusion map, which was superimposed onto the laparoscopic real-time video to obtain FLER, as described previously ${ }^{11-14}$ (Figure 2).

\section{Analysis of apillary lactate levels}

Using the level of local capillary lactate (LCL) as the primary outcome, a sample size calculation was performed based on previous work from our group using a similar experimental model. Using a superiority design, 50 simultaneously analyzed spots with both techniques were required to have a $90 \%$ chance of detecting a difference in the primary outcome measure at a statistical significance of 5\%. Because an ischemic bowel loop was created in each animal and each loop contained 9 regions of interest (ROIs), 8 animals (72 ROIs) in total were included. LCL served as "ground-truth" metric to compare the performances of FLER and HYPER. A strip-based, portable lactate analyzer (EDGE®, ApexBio, Taipei, Taiwan; measuring range: $1.1-22.2 \mathrm{mmol} / \mathrm{L}$ ) was used to assess lactate levels on blood punctured from the serosal capillary vessels in correspondence with each identified ROI. The order of sampling was randomized. The robustness of LCL to quantify the perfusion of the gastrointestinal tract has been validated previously ${ }^{11-14,17,20,21}$.

Systemic lactatemia was measured on blood samples obtained by puncturing capillary vessels on the pigs' snout and LCLs were normalized to the systemic values.

\section{Operative set-up and experimental workflow}

A laparotomy was performed, and a $10 \mathrm{~cm}$ ischemic segment was created in the proximal jejunum by dividing the arcade branches (Figure 3). After $30 \mathrm{~min}$ of ischemia, HYPER was first performed, displaying the $\mathrm{StO}_{2}$ pseudo-color images onto the real-time laparoscopic video shown directly on the OR monitor. Nine contiguous ROIs, including perfused and ischemic areas, were placed randomly throughout the length of the bowel loop and displayed in 
augmented reality. Immediately afterwards, ICG was administered intravenously, and FLER

was obtained and simultaneously superimposed onto HYPER (Figure 2). As next step, LCLs were obtained in randomized order at the 9 ROIs displayed in augmented reality .

The HSI images were obtained in approximately $6 \mathrm{~s}$ for each acquisition with the $\mathrm{StO}_{2}$ cartography was provided instantaneously. The time required to position the ROIs and obtain HYPER was approximately $1 \mathrm{~min}$. The delay between ICG administration in order to perform FA and superimposition of FLER onto HYPER was approximately of $2 \mathrm{~min}$. Therefore, the two imaging techniques were performed with a time interval of 1 min. The delay between HYPER completion and lactate sampling was of roughly $3 \mathrm{~min}$.

In order to minimize any bias possibly generated by motion or light artefacts, breathing was stopped, and external light interference was prevented during the time required to obtain both imaging acquisitions ( $6 \mathrm{~s}$ for HSI and $40 \mathrm{sec}$ for FA).

\section{Prediction of local capillary lactates from FLER TTP and HSI StO2}

The relationship between LCLs and FLER TTP and HSI $\mathrm{StO}_{2}$ respectively, followed an exponential trend, which was modeled by an exponential regression analysis. This technique allowed us to generate a prediction algorithm of the LCL values from the corresponding FLER TTP and $\mathrm{HSI}-\mathrm{StO}_{2}$. The following fitting functions were found:

1) based on the FLER TTP:

predicted $L C L=e^{0.01 * T T P-0.553}+$ SystemicLactates

2) based on $\mathrm{HSI}-\mathrm{StO}_{2}$ :

predictedLCL $=e^{-0.049 * \mathrm{StO}_{2}+2.22}+$ SystemicLactates 
In order to validate the prediction models, a mean square minimization of the objective function $\exp (-a x+b)$ was performed on normalized LCLs using the Scipy, Python library ${ }^{22}$. The prediction functions were obtained from the whole datasets, because no significance difference in terms of error prediction could be observed when performing a leave-one-(pig) out crossvalidation.

The accuracy of both models, indicated as the absolute difference between the sampled LCLs and the predicted LCL values of both prediction models, was computed on the whole data by the corresponding exponential equation.

\section{Statistical analysis}

Data are shown as mean \pm SD unless indicated otherwise. Statistics were performed using the Python Scikit-learn library ${ }^{23}$. A Pearson's test was used to compare variables showing a linear relationship. Spearman correlation and exponential regression were used to investigate variables presenting a non-linear relationship. A Wilcoxon test was performed for paired comparison of the lactate prediction algorithms (based on HYPER and on FLER), because a non-Gaussian data distribution was observed. A $p$ value $<0.05$ was considered statistically significant. 


\section{Results}

Using the enhanced reality, we were able to identify precisely the ROIs on the bowel loops $(n=72)$ as demonstrated by both HSI-StO2 and FLER TTP, thereby allowing accurate sampling of serosal capillary blood to measure LCL. Mean systemic lactate concentration was $2.63 \pm 2.85 \mathrm{mmol} / \mathrm{L}$. Considering all the ROIs, the mean LCL concentration was $4.67 \pm 4.34$ $\mathrm{mmol} / \mathrm{L}$, the mean normalized LCL value was $2.03 \pm 2.44 \mathrm{mmol} / \mathrm{L}$, the mean $\mathrm{StO}_{2}$ value was $45.9 \pm 18.6 \%$ and the mean TTP was $10.3 \pm 9.4$ seconds.

\section{Correlation between FLER TTP and HSI-StO2}

The Pearson analysis between FLER TTP and HSI-StO2 in correspondence with the same ROIs gave a $R=-0.66(p<0.0001)($ Figure 4 a).

\section{Correlation between LCL and TTP / LCL and StO2}

The Spearman correlation between LCLs and FLER TTP gave a $\mathrm{R}=0.40(\mathrm{p}=0.001)$, and a $\mathrm{R}=-$ $0.62(\mathrm{p}<0.0001)$ between LCLs and HSI-StO2. Both correlations produced 7 outliers (Figure 4 b-c), including only completely ischemic ROIs, with LCL > $6 \mathrm{mmol} / \mathrm{l}$. These 7 outliers were excluded from the analysis of the accuracy of the LCL prediction models reported below.

\section{LCL prediction based on FLER TTP}

The mean error of the lactate prediction model was $0.95 \pm 0.74 \mathrm{mmol} / \mathrm{L}$. The median error was $0.68 \mathrm{mmol} / \mathrm{L}$, and $95 \%$ of the errors occurred for LCLs $<2.33 \mathrm{mmol} / \mathrm{L}$.

\section{LCL prediction based on HSI-StO2}

The lactate prediction model showed a mean error of $0.65 \pm 0.59 \mathrm{mmol} / \mathrm{L}$, with a median error of $0.43 \mathrm{mmol} / \mathrm{L}, 95 \%$ of the errors occurred for LCLs $<2 \mathrm{mmol} / \mathrm{L}$.

\section{Comparison between FLER-based and HSI-based lactate prediction models}

The Wilcoxon test showed a difference between the two prediction models $(\mathrm{p}<0.0001)$, with HSI proving a better lactate prediction performance (Figure 5). 
1

2

3

4

5

9

10

11

12

13

14

15

16

17

18

19

20

21

22

23

24

25

26

27

28

29

30

31

32

33

34

35

36

37

38

39

40

41

42

43

44

45

46

47

48

49

50

51

52

53

54

55

56

57

58

59

60

61

62

63

64

65 


\section{Discussion}

The results of the present study show that both FLER and HYPER give virtually real-time information, and the metrics used by both quantitative methods, TTP and $\mathrm{StO}_{2}$, are correlate well. Additionally, both parameters have a statistically significant exponential relationship with the LCLs. A fitting function between LCL and both the FLER TTP and HSI-StO 2 was found and provided two LCL prediction models. Both prediction models were less accurate for LCLs $>6 \mathrm{mmol} / \mathrm{L}$. As a result, a threshold below this value was set which led to excluding a total of 7 ROIs which were definitely outliers (Figure 4 b-c). This correction lead to a substantially improved precision of the prediction algorithms. These findings are in line with the results of our prior study, in which HYPER provided a significantly better accuracy of the LCL prediction for $\mathrm{StO}_{2}$ values $>30 \%{ }^{17}$. In the present study, all outlying ROIs (LCL>6mmol/L) showed that $\mathrm{StO}_{2}$ was $<30 \%$. Likewise, it is clinically irrelevant to predict the LCL in ROIs precisely which are frankly ischemic based on a quantitative optical imaging analysis and which are often identifiable as non-perfused areas to the naked eye. Both prediction models showed high accuracy in discriminating marginally perfused areas, which are often difficult to identify under white light. The precise identification of those areas is important not only to assess the perfusion of gastrointestinal anastomoses, but also to evaluate the bowel viability after mesenteric ischemia. As outlined by Kougias et al., second-look laparotomies with additional bowel resections are required in $57 \%$ of cases presenting with mesenteric ischemia ${ }^{24}$. Therefore, noninvasive optical imaging tools, like FLER or HYPER, could improve the intraoperative decision-making process. We must stress, however, that, the threshold of those imaging modalities representing the "point of no return", irreversible ischemia, has yet to be identified and is currently under investigation.

The prediction model based on the FLER TTP was less accurate when compared to the model based on the $\mathrm{HSI} \mathrm{StO}_{2}$. A possible explanation could be the short ischemia period (30 minutes) 
chosen in the present set-up. During this time, macroscopic signs of tissue injury are rarely

found. there are, however, intracellular changes which decrease mitochondrial activity. As a result, decreases in $\mathrm{O}_{2}$ and increases in LCL production already occur ${ }^{14}$. The HSI-detected $\mathrm{StO}_{2}$ seems to better mirror those intracellular metabolic changes as compared to FLER, which finally measures a macroscopic phenomenon such as the serosal surface blood flow.

FA is a well-established intraoperative method to assess gastrointestinal perfusion and is relatively easy to integrate in the surgical workflow ${ }^{9}$, but there is still no consensus on the impact of FA on decreasing the incidence of anastomotic leakage, and the results of randomized clinical trials are awaited ${ }^{25}$. Two factors affect the performance of FA and should be controlled. The first factor is the progressive diffusion over time of the fluorophore through the serosal capillary network into the margins of the ischemic zones, leading to an overestimation of the vascularized area. The second factor is the quadratic inverse relationship between the targetendoscope distance and fluorescence intensity ${ }^{11,14}$. When distance is not controlled and/or fluorescence intensity is not normalized with a calibration system, the presence of a fluorescent signal in the tissue is not representative of the true perfusion. To overcome such interpretation biases, a software-based quantitative FA was introduced based on the analysis of the dynamics of the fluorescent signal ${ }^{11,14,19}$. Wada et al. successfully created an algorithm of quantitative fluorescence analysis during clinical colorectal $\operatorname{cases}^{19}$. The authors used a similar algorithm of evaluating the fluorescence dynamics ${ }^{11}$; however, unlike FLER, the enhanced information (virtual perfusion cartography) was not superimposed onto real-time images. FLER is also currently being in a cluated trial $^{26 \text {, }}$ ${ }^{27}$ (https://clinicaltrials.gov/ct2/show/NCT02626091) with promising early results in terms of correlation with surrogate markers of perfusion and prediction of anastomotic complications. HSI has been used for decades in various industrial sectors or for remote sensing ${ }^{28}$. Recently, HSI was used successfully in medical and surgical applications, including identification of 
various tumors ${ }^{28}$, intraoperative parathyroid recognition ${ }^{29}$, and real-time quantitative assessment of perfusion during gastrointestinal operations ${ }^{30-32}$.

Compared to fluorescence imaging, HSI provides a greater amount of information and is able to quantify several tissue components, including the content of water, oxygen, and hemoglobin. Additionally, a remarkable advantage when compared to FA is that HSI does not require the use of any contrast agents; thus, HSI is an optical, contrast-free, non-destructive in vivo "biopsy" of the operative field, allowing one to virtually discriminate anatomic structures and characterize certain aspects of tissue physiology. The large HSI-generated datasets, however, require complex data processing algorithms in order to extrapolate useful discriminative features from the spectral curves. The TIVITA® HSI system has a built-in software which generates pseudo-color images by quantifying $\mathrm{StO}_{2}{ }^{29-31}$. We used a proprietary software solution for the superimposing of the $\mathrm{HSI} \mathrm{StO}_{2}$ images onto real-time video images in order to create an enhanced reality surgical scene (HYPER) and to identify precisely the small ROIs on the bowel surface.

To the best of our knowledge, the present study is the first comparison between HSI and rtancwe quantitative FA to assess bowel perfusion. The importance of our study lies in its robust design and methodology. Limitations of this study lie in the fact that the results are obtained in controlled experimental conditions of early ischemia (30 minutes) and the clinical application of HYPER has not been demonstrated yet. Furthermore, although the acquisitions of the two imaging systems occurred virtually simultaneously (1-minute delay), the study draws on comparison between a minimally invasive NIR camera and an open-surgery HSI camera. In this setting, HYPER displayed a better overall accuracy. While this difference was statistically significant, it remains unknown if this difference will prove to be clinically important.

Currently, HSI systems are limited by the lack of an adequately fast video rate and by the lack of a system for minimally invasive surgery. Some authors have proposed endoscopic HSI 
systems $^{33,34}$. but the spatial resolution and speed of the data processing are still limited enough to allow its use in our daily surgical practice.

Nevertheless, HYPER represents an attractive tool to perform a non-invasive, contrast-free, real-time and accurate, intraoperative perfusion quantification.

As next step, a trial to assess the accuracy of HYPER intraoperatively is currently being designed.

\section{Acknowledgments}

Authors are grateful to Guy Temporal, Christopher Burel, and Camille Goustiaux, professionals in medical English proofreading, for their valuable help in revising the manuscript.

COI/DISCLOSURES: Jacques Marescaux is the President of both IRCAD and IHU Strasbourg Institutes, which are partly funded by KARL STORZ, Siemens, and Medtronic. Lee Swanström is the Scientific Director of the IHU and Michele Diana is the recipient of the ELIOS grant. Manish Chand has received fees from Stryker Endoscopy for his work as a consultant for fluorescent angiography, outside of this work. The remaining authors have no conflicts of interest or financial ties to disclose.

FUNDING/SUPPORT: This work was funded by the ARC Foundation through the ELIOS (Endoscopic Luminescent Imaging for precision Oncologic Surgery) grant 


\section{References:}

1. Campbell C, Reames MK, Robinson M, Symanowski J, Salo JC. Conduit Vascular Evaluation is Associated with Reduction in Anastomotic Leak After Esophagectomy. J Gastrointest Surg. 2015;19:806-12.

2. Karliczek A, Benaron DA, Baas PC, Zeebregts CJ, Wiggers T, van Dam GM. Intraoperative assessment of microperfusion with visible light spectroscopy for prediction of anastomotic leakage in colorectal anastomoses. Colorectal Dis.12:1018-25.

3. Urbanavicius L, Pattyn P, de Putte DV, Venskutonis D. How to assess intestinal viability during surgery: A review of techniques. World J Gastrointest Surg. 2011;3:59-69.

4. Karliczek A, Harlaar NJ, Zeebregts CJ, Wiggers T, Baas PC, van Dam GM. Surgeons lack predictive accuracy for anastomotic leakage in gastrointestinal surgery. Int J Colorectal Dis. 2009;24:569-76.

5. Kudszus S, Roesel C, Schachtrupp A, Hoer JJ. Intraoperative laser fluorescence angiography in colorectal surgery: a noninvasive analysis to reduce the rate of anastomotic leakage. Langenbecks Arch Surg. 2010;395:1025-30.

6. Jafari MD, Wexner SD, Martz JE, McLemore EC, Margolin DA, Sherwinter DA, et al. Perfusion assessment in laparoscopic left-sided/anterior resection (PILLAR II): a multiinstitutional study. J Am Coll Surg. 2015;220:82-92 e1.

7. Ris F, Liot E, Buchs NC, Kraus R, Ismael G, Belfontali V, et al. Multicentre phase II trial of near-infrared imaging in elective colorectal surgery. Br J Surg. 2018;105:1359-67.

8. De Nardi P, Elmore U, Maggi G, Maggiore R, Boni L, Cassinotti E, et al. Intraoperative angiography with indocyanine green to assess anastomosis perfusion in patients undergoing laparoscopic colorectal resection: results of a multicenter randomized controlled trial. Surg Endosc. 2019.

9. Blanco-Colino R, Espin-Basany E. Intraoperative use of ICG fluorescence imaging to reduce the risk of anastomotic leakage in colorectal surgery: a systematic review and meta-analysis. Tech Coloproctol. 2018;22:15-23.

10. Vallance A, Wexner S, Berho M, Cahill R, Coleman M, Haboubi N, et al. A collaborative review of the current concepts and challenges of anastomotic leaks in colorectal surgery. Colorectal Dis. 2017;19:01-012.

11. Diana M, Agnus V, Halvax P, Liu YY, Dallemagne B, Schlagowski Al, et al. Intraoperative fluorescence-based enhanced reality laparoscopic real-time imaging to assess bowel perfusion at the anastomotic site in an experimental model. Br J Surg. 2015;102:e16976.

12. Diana M, Dallemagne B, Chung H, Nagao Y, Halvax P, Agnus V, et al. Probe-based confocal laser endomicroscopy and fluorescence-based enhanced reality for real-time assessment of intestinal microcirculation in a porcine model of sigmoid ischemia. Surg Endosc. 2014;28:3224-33.

13. Diana M, Halvax P, Dallemagne B, Nagao Y, Diemunsch P, Charles AL, et al. Real-time navigation by fluorescence-based enhanced reality for precise estimation of future anastomotic site in digestive surgery. Surg Endosc. 2014;28:3108-18.

14. Diana M, Noll E, Diemunsch P, Dallemagne B, Benahmed MA, Agnus V, et al. Enhancedreality video fluorescence: a real-time assessment of intestinal viability. Ann Surg. 2014;259:700-7. 
15. Grambow E, Dau M, Holmer A, Lipp V, Frerich B, Klar E, et al. Hyperspectral imaging for Microvasc Res. 2018;116:64-70.

16. Holmer A, Marotz J, Wahl P, Dau M, Kammerer PW. Hyperspectral imaging in perfusion and wound diagnostics - methods and algorithms for the determination of tissue parameters. Biomed Tech (Berl). 2018;63:547-56.

17. Barberio $M$, Longo F, Fiorillo C, Seeliger B, Mascagni P, Agnus V, et al. HYPerspectral Enhanced Reality (HYPER): a physiology-based surgical guidance tool. Surg Endosc. 2019.

18. Kilkenny C, Browne W, Cuthill IC, Emerson M, Altman DG, Group NCRRGW. Animal research: reporting in vivo experiments: the ARRIVE guidelines. J Gene Med. 2010;12:561-3.

19. Wada T, Kawada K, Takahashi R, Yoshitomi M, Hida K, Hasegawa S, et al. ICG fluorescence imaging for quantitative evaluation of colonic perfusion in laparoscopic colorectal surgery. Surg Endosc. 2017;31:4184-93.

20. Diana M, Noll E, Charles AL, Diemunsch P, Geny B, Liu YY, et al. Precision real-time evaluation of bowel perfusion: accuracy of confocal endomicroscopy assessment of stoma in a controlled hemorrhagic shock model. Surg Endosc. 2017;31:680-91.

21. Diana M, Noll E, Diemunsch P, Moussallieh FM, Namer IJ, Charles AL, et al. MetabolismGuided Bowel Resection: Potential Role and Accuracy of Instant Capillary Lactates to Identify the Optimal Resection Site. Surg Innov. 2015;22:453-61.

22. Virtanen P, Gommers R, Oliphant TE, Haberland M, Reddy T, Cournapeau D, et al. SciPy 1.0--Fundamental Algorithms for Scientific Computing in Python. arXiv preprint arXiv:190710121. 2019.

23. Pedregosa F, Varoquaux G, Gramfort A, Michel V, Thirion B, Grisel O, et al. Scikit-learn: Machine learning in Python. Journal of machine learning research. 2011;12:2825-30.

24. Kougias P, Lau D, El Sayed HF, Zhou W, Huynh TT, Lin PH. Determinants of mortality and treatment outcome following surgical interventions for acute mesenteric ischemia. Journal of vascular surgery. 2007;46:467-74.

25. Armstrong G, Croft J, Corrigan N, Brown JM, Goh V, Quirke P, et al. IntAct: intraoperative fluorescence angiography to prevent anastomotic leak in rectal cancer surgery: a randomized controlled trial. Colorectal Dis. 2018;20:0226-034.

26. Seeliger B, Barberio M, D'Urso A, Agnus V, Longo F, Mascagni $P$, et al. Fluorescence in rectal cancer surgery. Annals of Laparoscopic and Endoscopic Surgery. 2018;3:47

27. Mascagni $P$, Longo F, Barberio M, Seeliger B, Agnus V, Saccomandi $P$, et al. New intraoperative imaging technologies: Innovating the surgeon's eye toward surgical precision. J Surg Oncol. 2018;118:265-82.

28. Lu G, Fei B. Medical hyperspectral imaging: a review. J Biomed Opt. 2014;19:10901.

29. Barberio M, Maktabi M, Gockel I, Rayes N, Jansen-Winkeln B, Köhler H, et al. Hyperspectral based discrimination of thyroid and parathyroid during surgery. Current Directions in Biomedical Engineering2018. p. 399.

30. Jansen-Winkeln B, Maktabi M, Takoh JP, Rabe SM, Barberio $M$, Kohler $\mathrm{H}$, et al. [Hyperspectral imaging of gastrointestinal anastomoses]. Chirurg. 2018;89:717-25.

31. Kohler $H$, Jansen-Winkeln B, Maktabi M, Barberio M, Takoh J, Holfert $N$, et al. Evaluation of hyperspectral imaging (HSI) for the measurement of ischemic conditioning effects of the gastric conduit during esophagectomy. Surg Endosc. 2019. 
32. Jansen-Winkeln B, Holfert N, Kohler H, Moulla $\mathrm{Y}$, Takoh JP, Rabe SM, et al. Determination of the transection margin during colorectal resection with hyperspectral imaging (HSI). Int J Colorectal Dis. 2019;34:731-9.

33. Clancy NT, Arya S, Stoyanov D, Singh M, Hanna GB, Elson DS. Intraoperative measurement of bowel oxygen saturation using a multispectral imaging laparoscope. Biomed Opt Express. 2015;6:4179-90.

34. Zuzak KJ, Naik SC, Alexandrakis G, Hawkins D, Behbehani K, Livingston E. Intraoperative bile duct visualization using near-infrared hyperspectral video imaging. Am J Surg. 2008;195:491-7.

\section{Figures Legends}

Fig.1: TTP-representation. Graphic representation of the time-to-peak in well-perfused (blue) and ischemic (green) areas, in which very different maximum fluorescent intensity peaks (Fmax) were reached. TTP is the time required (in seconds) for the intensity curve to go from the first to the last quartile of fluorescent intensity over time. As represented, TTP is less for the perfused zone when compared to the ischemiczone.

Fig.2: Enhanced reality modalities overlays. Ischemic small bowel model displayed under white light (A), with ICG reference card visible in the center of the bowel loop. FLER (B) with superimposing of the fluorescence-based quantitative perfusion cartography. HYPER (C) generated through the superimposing of the $\mathrm{StO}_{2}$ pseudo-color image captured with HSI. (D) shows the warping of FLER and HYPER simultaneously, over the real-time video captured with the NIR-laparoscopic camera during the white light mode. The selected ROIs are precisely identifiable in the operative scene and LCLs were sampled in correspondence with them.

Figure 3: Operative set-up. The ischemic loop is placed on top of the calibration chessboard in order to facilitate the accurate superimposing of images obtained from cameras with different angles. The laparoscopic camera (arrow) and the HSI system (arrowhead) are placed in order to capture the operative scene from different angles but with the same picture orientation. 
Figure 4: TTP/StO $\mathrm{S}_{2}$ correlation and LCL regression analysis. (A) Visual representation of Pearson's correlation between HSI-StO2 and FLER TTP. The exponential regression correlation between LCLs and both FLER TTP (B) and HSI-StO2 (C) is shown. Seven datasets of highly ischemic regions (shadowed points) were excluded from the regression analysis, because using both imaging modalities, the LCL prediction models were less accurate when the LCL was $>6 \mathrm{mmol} / \mathrm{L}$, as highlighted in the graph.

Figure 5: Cumulative error of lactate prediction. Overall precision in predicting LCL was statistically significantly greater when using the HSI-StO2 (black line) when compared to FLER TTP (red line). 
TOC Statement- 20191302

FLuorescence-based Enhanced Reality (FLER) is a software that provides quantitative fluorescence angiography (FA), by computing the fluorescence intensity time-to-peak (TTP) after intravenous injection of indocyanine green (ICG). Hyperspectral imaging (HIS) is a contrast-free, optical imaging modality, which measures tissue oxygenation (StO2). The importance of this report is that bowel perfusion was clearly quantified using HSI and FLER in a pig model. 
TABLE 1. RAW DATA

\begin{tabular}{|c|c|c|c|c|c|}
\hline $\begin{array}{c}\text { Pig } \\
\text { number }\end{array}$ & $\begin{array}{c}\mathrm{HSI}^{-\mathrm{StO}_{2}} \\
(\%)\end{array}$ & $\begin{array}{c}\text { FLER-TPP } \\
\text { (sec.) }\end{array}$ & $\begin{array}{c}\mathrm{LCL} \\
(\mathrm{mmol} / \mathrm{L})\end{array}$ & $\begin{array}{l}\text { Systemic } \\
\text { Lactates } \\
\text { (mmol/L) }\end{array}$ & $\begin{array}{c}\text { Normalized } \\
\text { Lactates } \\
\text { (mmol/L) }\end{array}$ \\
\hline 1 & 75 & 4 & 1,1 & 0,4 & 0,7 \\
\hline 1 & 69 & 3,8 & 0,4 & 0,4 & 0 \\
\hline 1 & 80 & 4,6 & 1,5 & 0,4 & 1,1 \\
\hline 1 & 70 & 4,6 & 1,1 & 0,4 & 0,7 \\
\hline 1 & 47 & 12,2 & 3 & 0,4 & 2,6 \\
\hline 1 & 16 & 40 & 5 & 0,4 & 4,6 \\
\hline 1 & 19 & 11,6 & 4 & 0,4 & 3,6 \\
\hline 1 & 37 & 12,6 & 2,1 & 0,4 & 1,7 \\
\hline 1 & 54 & 6,6 & 1,3 & 0,4 & 0,9 \\
\hline 2 & 62 & 3 & 2,2 & 2,2 & 0 \\
\hline 2 & 67 & 3,8 & 2,4 & 2,2 & 0,2 \\
\hline 2 & 72 & 4,4 & 2,4 & 2,2 & 0,2 \\
\hline 2 & 45 & 6,8 & 2,8 & 2,2 & 0,6 \\
\hline 2 & 28 & 14,6 & 5,4 & 2,2 & 3,2 \\
\hline 2 & 20 & 5,8 & 5,5 & 2,2 & 3,3 \\
\hline 2 & 44 & 12 & 2,7 & 2,2 & 0,5 \\
\hline 2 & 69 & 7,4 & 2,3 & 2,2 & 0,1 \\
\hline 2 & 80 & 4 & 2,3 & 2,2 & 0,1 \\
\hline 3 & 37 & 15,6 & 3,8 & 3,7 & 0,1 \\
\hline 3 & 45 & 16,2 & 4,4 & 3,7 & 0,7 \\
\hline 3 & 46 & 16,2 & 3,8 & 3,7 & 0,1 \\
\hline 3 & 32 & 17,2 & 4,8 & 3,7 & 1,1 \\
\hline 3 & 18 & 15,4 & 7,3 & 3,7 & 3,6 \\
\hline 3 & 15 & 8,8 & 10,4 & 3,7 & 6,7 \\
\hline 3 & 35 & 16,8 & 6,1 & 3,7 & 2,4 \\
\hline 3 & 48 & 17,4 & 3,7 & 3,7 & 0 \\
\hline 3 & 47 & 16,2 & 4 & 3,7 & 0,3 \\
\hline 4 & 74 & 4 & 2,2 & 2 & 0,2 \\
\hline 4 & 56 & 4 & 2,3 & 2 & 0,3 \\
\hline 4 & 71 & 4,2 & 2 & 2 & 0 \\
\hline 4 & 58 & 8,4 & 2,6 & 2 & 0,6 \\
\hline 4 & 33 & 10,6 & 4,2 & 2 & 2,2 \\
\hline 4 & 29 & 12,2 & 5,2 & 2 & 3,2 \\
\hline 4 & 37 & 9,6 & 4,3 & 2 & 2,3 \\
\hline 4 & 57 & 5,4 & 3 & 2 & 1 \\
\hline 4 & 76 & 4,4 & 2 & 2 & 0 \\
\hline 5 & 59 & 2,2 & 2,1 & 2 & 0,1 \\
\hline 5 & 58 & 2,2 & 2 & 2 & 0 \\
\hline 5 & 44 & 5,2 & 5 & 2 & 3 \\
\hline 5 & 28 & 13,4 & 8,2 & 2 & 6,2 \\
\hline 5 & 23 & 4,2 & 8,7 & 2 & 6,7 \\
\hline 5 & 46 & 12,6 & 4,4 & 2 & 2,4 \\
\hline 5 & 49 & 3,8 & 3,1 & 2 & 1,1 \\
\hline 5 & 57 & 2,8 & 2 & 2 & 0 \\
\hline 5 & 67 & 2,6 & 2,7 & 2 & 0,7 \\
\hline 6 & 59 & 3 & 0,8 & 0,8 & 0 \\
\hline 6 & 55 & 3,2 & 1,3 & 0,8 & 0,5 \\
\hline 6 & 57 & 3,4 & 1,2 & 0,8 & 0,4 \\
\hline
\end{tabular}




\begin{tabular}{|r|r|r|r|r|r|}
\hline & 32 & 5,7 & 3,1 & 0,8 & 2,3 \\
\hline 6 & 21 & 11,6 & 3,3 & 0,8 & 2,5 \\
\hline 6 & 33 & 5 & 2,8 & 0,8 & 2 \\
\hline 6 & 47 & 3,4 & 1,4 & 0,8 & 0,6 \\
\hline 6 & 65 & 3,6 & 1,6 & 0,8 & 0,8 \\
\hline 6 & 53 & 3,4 & 1,2 & 0,8 & 0,4 \\
\hline 7 & 51 & 3,4 & 0,4 & 0,4 & 0 \\
\hline 7 & 53 & 3,6 & 3,1 & 0,4 & 2,7 \\
\hline 7 & 18 & 8,6 & 8,6 & 0,4 & 8,2 \\
\hline 7 & 50 & 5,2 & 3,3 & 0,4 & 2,9 \\
\hline 7 & 71 & 3,6 & 3 & 0,4 & 2,6 \\
\hline 7 & 74 & 3,2 & 2,1 & 0,4 & 1,7 \\
\hline 7 & 58 & 2,8 & 2,5 & 0,4 & 2,1 \\
\hline 7 & 54 & 5,4 & 2,5 & 0,4 & 2,1 \\
\hline 7 & 25 & 10,8 & 4,4 & 0,4 & 4 \\
\hline 8 & 29 & 19,2 & 9,6 & 9,6 & 0 \\
\hline 8 & 26 & 8,4 & 12,6 & 9,6 & 3 \\
\hline 8 & 26 & 26 & 12,6 & 9,6 & 3 \\
\hline 8 & 20 & 40 & 23 & 9,6 & 13,4 \\
\hline 8 & 23 & 40 & 11,8 & 9,6 & 2,2 \\
\hline 8 & 24 & 22,6 & 12,2 & 9,6 & 2,6 \\
\hline 8 & 28 & 25,4 & 17 & 9,6 & 7,4 \\
\hline 8 & 30 & 20 & 11,7 & 9,6 & 2,1 \\
\hline 8 & 25 & 40 & 17,5 & 9,6 & 7,9 \\
\hline
\end{tabular}

